\title{
A review of the shore-fly genus Ephydra Fallén, 1810 (Diptera: Ephydridae) of Russia
}

\section{Обзор видов мух-береговушек рода Еphydra Fallén, 1810 (Diptera: Ephydridae) фауны России}

\author{
M.G. Krivosheina ${ }^{1,3}$, A.L. Ozerov ${ }^{2}$ \\ М.Г. Кривошеина ${ }^{1,3}$, А.А. Озеров ${ }^{2}$
}

\author{
${ }^{1}$ A.N. Severtsov Institute of Ecology and Evolution, Russian Academy of Sciences, 119071 Moscow, Russia. \\ E-mail: kriv2260@rambler.ru \\ 'Институт проблем экологии и эволюции им. А.Н. Северцова РАН, Ленинский проспект, 33, Москва 119071, Россия. \\ ${ }^{2} Z$ oological Museum, Moscow Lomonosov State University, Bol'shaya Nikitskaya 2, Moscow 125009, Russia. \\ E-mail: ozerov2455@rambler.ru \\ 2Зоологический музей, Московский государственный университет им. М.В. Ломоносова, Большая Никитская ул., 2, Москва \\ 125009, Россия. \\ ${ }^{3}$ corresponding author
}

KEY WORDS: Diptera, Ephydridae, Ephydra, new species, Russia, description, key.

КЛЮЧЕВЫЕ СЛОВА: Diptera, Ephydridae, Ephydra, новый вид, Россия, описание, определительная таблица.

ABSTRACT. The species composition and distribution of the shore-flies of the genus Ephydra Fallén, 1810 (Diptera, Ephydridae) of the fauna of Russia are completely revised for the first time. In total, 9 species were found: E. afghanica Dahl, 1961, E. attica Becker, 1896, E. glauca Meigen, 1830, E. japonica Miyagi, 1966, E. macellaria Egger, 1862, E. pseudomurina Krivosheina, 1983, E. riparia Fallén, 1813 , E. scholtzi Becker, 1896, and one new species, E. villosa sp.n. The new species belongs to the glauca species group and differs from E.glauca in the number of intra-alar setae and inwardly curved surstyli covered with long setae. It was found in Irkutsk Oblast. The data on the distribution of Ephydra species on the territory of Russia and the Palearctic Region are updated. E. attica is recorded from Kazakhstan, Uzbekistan, Tajikistan, Turkey and Morocco for the first time; E. glauca is newly recorded from Ukraine, Kazakhstan and Uzbekistan; likewise, the following represent new country records, E. macellaria for Kyrgyzstan, Tajikistan and Mongolia; E. pseudomurina for Ukraine, Azerbaijan, Georgia, Kazakhstan and Tajikistan; E. riparia for Kazakhstan and E. scholtzi for Tajikistan. A key to species found on the territory of Russia is compiled.

РЕЗЮМЕ. Впервые полностью ревизованы состав и распространение мух-береговушек рода Ephydra Fallén, 1810 (Diptera, Ephydridae) фауны России. Всего обнаружено 9 видов: E. afghanica
Dahl, 1961, E. attica Becker, 1896, E. glauca Meigen, 1830, E. japonica Miyagi, 1966, E. macellaria Egger, 1862, E. pseudomurina Krivosheina, 1983, E. riparia Fallén, 1813, E. scholtzi Becker, 1896, и один новый для науки вид E. villosa sp.n. Новый вид относится к группе видов glauca и отличается от E.glauca количеством интраалярных щетинок и загнутыми вовнутрь сурстилями, покрытыми длинными щетинками. Он найден в Иркутсткой области. Уточнены данные по распространению видов Ephydra на территории России и Палеарктики. E. attica впервые найден в Казахстане, Узбекистане, Таджикистане, Турции и Марокко; E. glauca - на Украине, в Казахстане и Узбекистане; E. macellaria - в Киргизии, Таджикистане и Монголии; E. pseudomurina - на Украине, в Азербайджане, Грузии, Казахстане и Таджикистане; E. riparia - в Казахстане и $E$. scholtzi - в Таджикистане. Составлена определительная таблица видов, встречающихся на территории России.

\section{Introduction}

The genus Ephydra currently includes 34 species of the world fauna [Mathis, Zatwarnicki, 1995; ElMoursy et al., 2006]. Their distribution across zoogeographic regions is uneven, with 19 species recorded from the Palaearctic Region, 12 species from the Nearctic Region, 4 species known from the Afrotropical Region, 1 species from the Oriental Region, 3 species

How to cite this article: Krivosheina M.G., Ozerov A.L. 2021. A review of the shore-fly genus Ephydra Fallén, 1810 (Diptera: Ephydridae) of Russia // Russian Entomol. J. Vol.30. No.3. P.345-360. doi: 10.15298/ rusentj.30.3.15 
from the Neotropical Region, and 1 species from the Australasian-Oceanian Region.

Most of the species are distributed in one zoogeographic region only; however, 6 species are known from the territory of two or more regions: Ephydra riparia Fallén, 1813 is the only Holarctic species; $E$. macellaria Egger, 1862 and E. flavipes (Macquart, 1844) are registered in the Palearctic and Afrotropical Regions, E. japonica Miyagi, 1966 - in the Palaearctic and Oriental Regions, E. packardi Wirth, 1971 - in the Nearctic and Neotropical Regions, and E. millbrae Jones, 1906 - in the Nearctic, Neotropical and AustralasianOceanian Regions [Mathis, Zatwarnicki, 1995].

For the territory of Russia, taking into account the current synonymy, 4 species have been previously indicated: E. scholtzi Becker, 1896, E. glauca Meigen, 1830, E. riparia, and E. macellaria [Nartshuk, 1970].

Subsequent studies made it possible to identify additional 5 species: E. afghanica Dahl, 1961, E. attica Becker, 1896, E. japonica, E. murina Wirth, 1975 and E. pseudomurina Krivosheina, 1983 [Krivosheina, 2010; Przhiboro, Shadrin, 2012].

The study of the collection materials of the Zoological Museum of Moscow State University (ZMUM) and the Zoological Institute of the Russian Academy of Sciences, St.-Petersburg (ZISP) made it possible to expand our understanding of the composition and the distribution of species of the genus Ephydra on the territory of Russia.

The genus Ephydra Fallén, 1810 belongs to the tribe Ephydrini [Mathis, Zatwarnicki, 1995]. Among the Palaearctic genera, it is closest to the genus Setacera Cresson, 1930 [Krivosheina, Ozerov, 2020].

These two genera are actually similar in habitus and differ from the other representatives of the tribe Ephydrini in the micropubescent or pectinate arista, in the presence of 5 pairs of dorsocentral setae, in straight claws, and in the absence of pulvilli. The essential distinction between the genera is the presence of 2 (Setacera) or 3 (Ephydra) fronto-orbital setae. The larvae of these genera are also similar, but the creeping welts in the representatives of Setacera are less developed, and these larvae are inactive and develop among floating algae, while the larvae of Ephydra actively move over the bottom of a water reservoir, periodically coming to the surface, and are attached to algae and other floating substrates immediately prior to pupation [Krivosheina, 1987, 2003]. In addition, the larvae of the genus Setacera develop in fresh- and saltish-water reservoirs, and those of the genus Ephydra, in saltywater reservoirs only, standing the salinity up to $250 \%$. The larvae of both genera feed on detritus and microscopic algae and bacteria.

At the present time the genus Ephydra is divided into two subgenera: Halephydra Wirth, 1971 with the only species E. gracilis Packard, 1871, distributed in Australasian/Oceanian, Nearctic and Neotropical Regions and Ephydra, differing from it mainly in developed cruciate interfrontal setae and subshining colora- tion [Mathis, Marinoni, 2016]. Besides Wirth [1975] and subsequent authors distinguished three species groups among Ephydra: bivittata, with the only palaearctic species $E$. bivittata Loew, 1860, not registered in Russia, glauca and riparia.

The representatives of the two species groups, glauca and riparia from the subgenus Ephydra, distributed in Russia are discussed in the present work. The list of species is given in alphabetic order. The differences between the two species groups are described in the key.

\section{Material and methods}

The specimens examined for this study are deposited in the Zoological Museum, Moscow State University, Russia (ZMUM) and Zoological Institute of Russian Academy of Sciences, St. Petersburg, Russia (ZISP).

The majority of original Russian geographical names are given in transliteration, but names of some large geographical regions follow these indicated in Google Earth software.

The data on distributions are cited as follows: Russia is in the first place, other countries of the world are given after a m-dash [-].The terminology used in the generic and species descriptions follows McAlpine [1981], Cumming \& Wood [2009], and Stuckenberg [1999].

Morphological structures used for illustrations were examined with a Nikon SMZ645 zoom stereomicroscope and then photographed using an eTREK DCM900 digital camera attached in place of an eyepiece of monocular microscope. Resulting batches of images were processed with CombineZP software, editing of stacked images was performed in Adobe Photoshop.

\section{Taxonomic part}

\section{Genus Ephydra Fallén, 1810}

Ephydra Fallén, 1810: 22 (feminine). Type species: Ephydra riparia Fallén, 1813, designated by Curtis, 1832.

DIAGNOSIS. Medium-sized flies, body length 4$5.5 \mathrm{~mm}$; face prominently convex and pubescent, oral margin with setae; 3 (rarely 4) lateroclinate frontoorbital and $5(2+3)$ dorsocentral setae; claws straight; pulvilli not developed.

DESCRIPTION. Shiny bluish-green metallic or more or less matt pollinose species with broad head and small eyes.

Head. Face strongly swollen with vertical lower portion, covered with hairs, oral margin with a row of strong vertical setae; clypeus hidden (Figs 1, 2). Frons flat, frontal vitta shiny metallic, with a pair of cruciate strong or weak interfrontal setae; 3-4 pairs of lateroclinate fronto-orbital, 1 pair of ocellar, pairs of strong outer vertical and inner vertical setae; postocular setae well developed (Fig. 1) or short and thin (Fig. 2). Antenna blackish, greyish dusted; arista short (very short) pubescent (Fig. 2) or pectinate on dorsal surface (Fig. 6). 

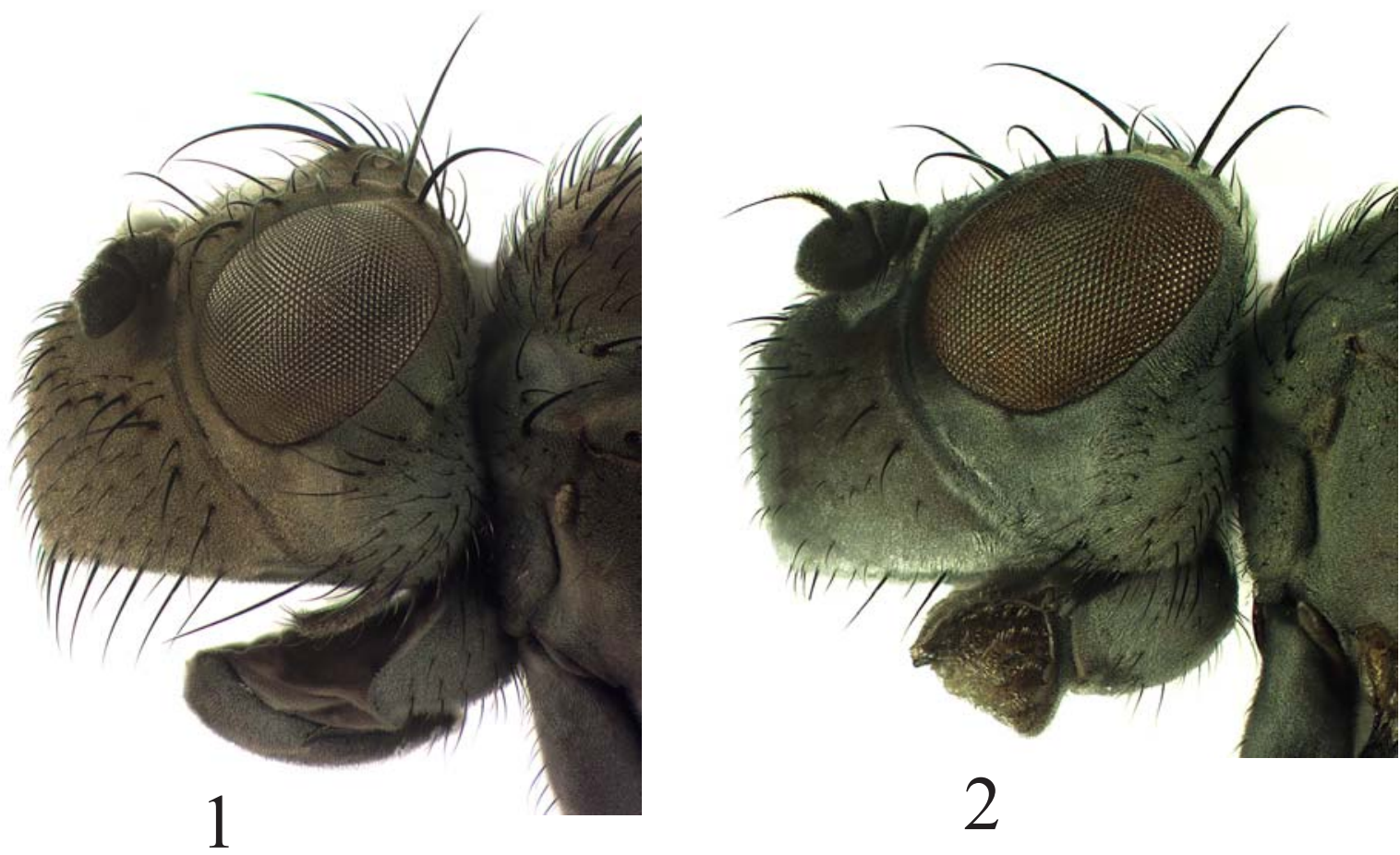

2

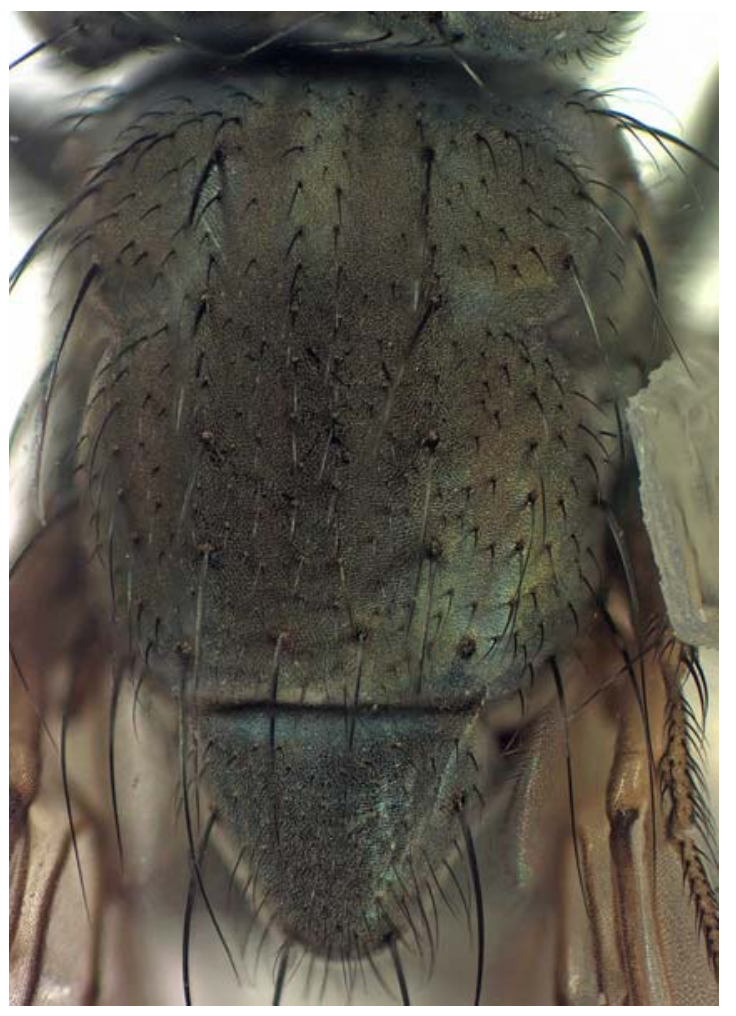

3

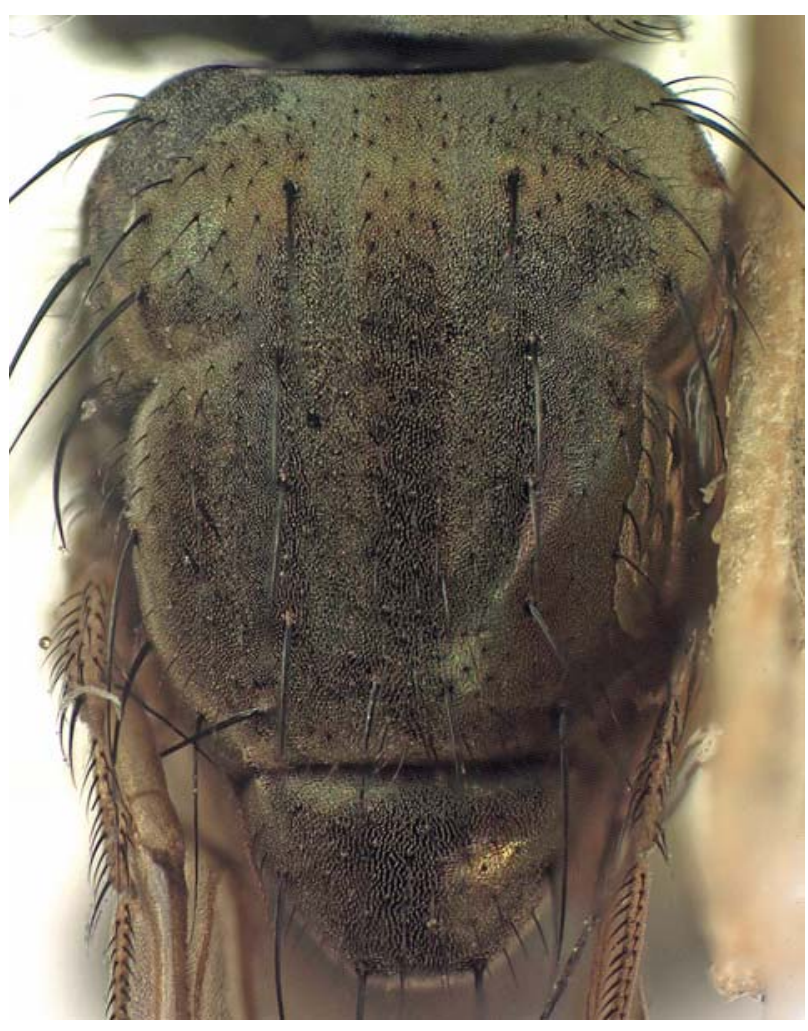

4

Figs 1-4. Head, lateral view $(1,2)$ and scutum $(3,4)$ of Ephydra spp.: 1,3 - E. glauca Meigen; $2-$ E. sholtzi Becker; $4-$ E. riparia Fallén.

Рис. 1-4. Голова, сбоку $(1,2)$ и среднеспинка $(3,4)$ Ephydra spp.: 1, 3 - E. glauca Meigen; 2 - E. sholtzi Becker; 4 - E. riparia Fallén. 


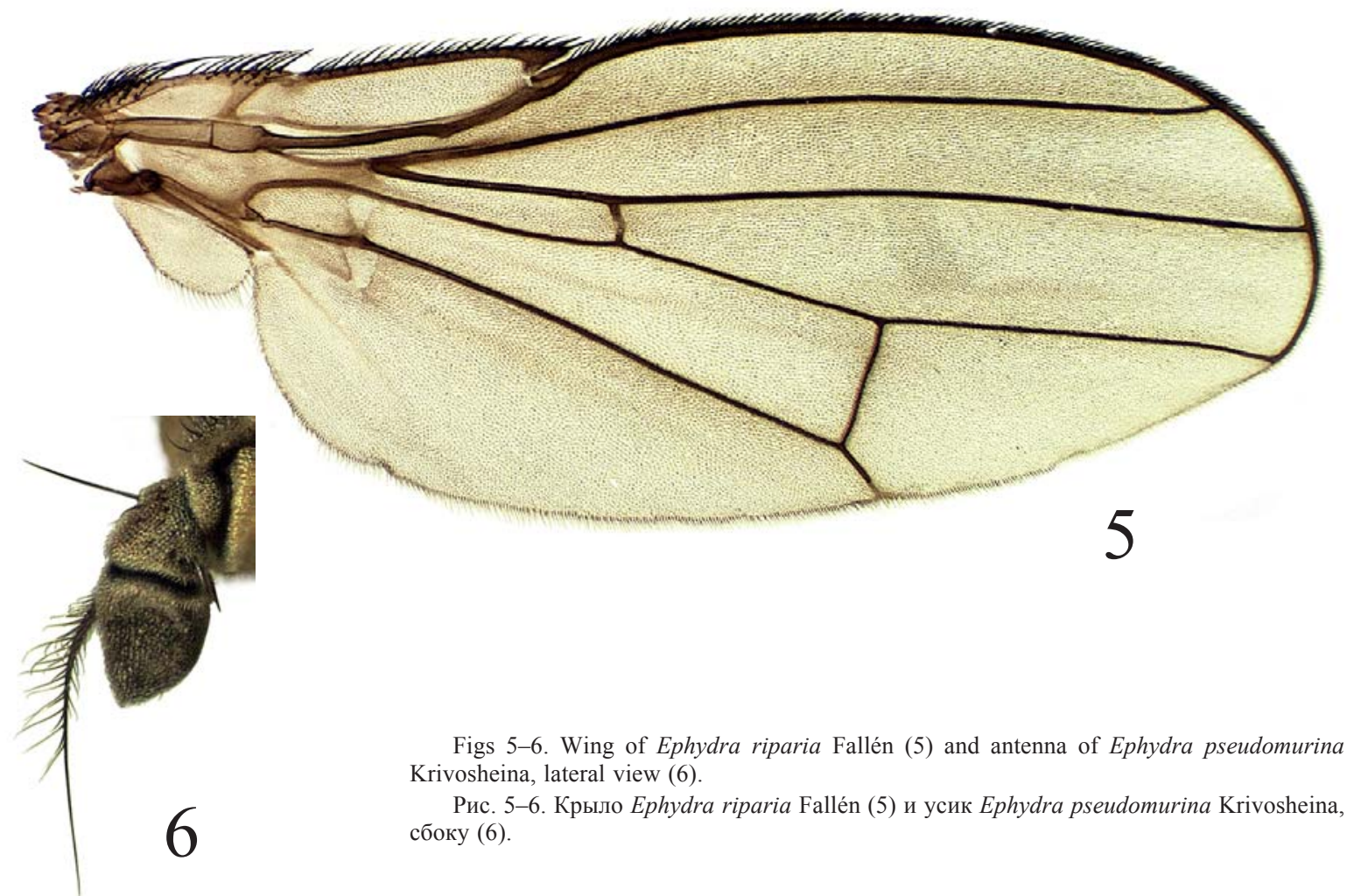

Thorax black, pollinose. Acrostichals setulose in many irregular rows at least in front of transverse suture (Fig. 4) or in two distinct rows (Fig. 3), dorsocentrals $2+3$, intra-alars $(0-2)+(0-2)$, supra-alars $2+1$, postpronotal 1 , notopleurals 2 , postalars 2 . Scutellum black, pollinose, with two pairs of strong setae. Anepisternum with 1 strong seta and a row of long hairs on posterir margin. Katepisternum with 1 strong seta in upper posterior corner.

Wing clear, with brownish tinge, without spots (Fig. 5). Calypters, margins of calypters and halteres yellow.

Abdomen black, pollinose. Structures of male terminalia considerably modified depending on species group (see below).

\section{Ephydra afghanica Dahl, 1961}

Figs 7, 16, 25, 34 .

afghanica Dahl, 1961: 87 (Ephydra). Type-locality: Dahlah, Qandahar (22 km N) (Afghanistan).

MATERIAL. Astrakhan' Oblast: Lake Baskunchak $\left(48.193^{\circ} \mathrm{N}\right.$ $\left.46.813^{\circ} \mathrm{E}\right), 2-4 . V .2010$, K.P. Tomkovich ( $2 \sigma^{7} \sigma^{7}$, ZMUM); Khakassia: salt lake Beljo $\left(54.65^{\circ} \mathrm{N} 90.18^{\circ} \mathrm{E}\right), 382 \mathrm{~m}, 1-3 . \mathrm{VII} .2011$, K.P. Tomkovich (1 $\sigma^{7}$, ZMUM).

ADDITIONAL MATERIAL. Armenia: Parakar $\left(40.164^{\circ} \mathrm{N} 44\right.$. 413ํㅡ), 11.IX.1962, V. Richter (1 $0^{7}$, ZISP); China: Kurlyk [= Lake Hurleg $\left.\mathrm{Hu}, 37.28^{\circ} \mathrm{N} 96.89^{\circ} \mathrm{E}\right]$, eastern Baingol, Caidam, V. 1895, coll. Roborowsky et Kozlov (1 $\bigcirc^{\top}$, ZISP); Kyrgyzstan: Lake Issyk-Kul', Rybach'e [=Balykchi] $\left(42.465^{\circ} \mathrm{N} 76.182^{\circ} \mathrm{E}\right), 1600 \mathrm{~m}$ 16.VIII.1969, K. Gorodkov (2 $\bigcirc^{7} \bigcirc^{7}$, ZISP); Turkmenistan: $30 \mathrm{~km}$ NW of Ashgabat $\left(38.164^{\circ} \mathrm{N} 57.960^{\circ} \mathrm{E}\right)$, 16.IV.1983, 18.IV.1983, M. Krivosheina (21 $0^{7} \sigma^{7}$, ZMUM); Ashgabat $\left(37.934^{\circ} \mathrm{N} 58.387^{\circ} \mathrm{E}\right)$, 15.XI.1983, N. Krivosheina (2 $\bigcirc^{7} \sigma^{7}$, ZMUM); Bayram-Ali (37. $\left.618^{\circ} \mathrm{N} 62.162^{\circ} \mathrm{E}\right), 18-19 . \mathrm{IV} .1930$, P. Bogush (1 $\sigma^{7}$, ZISP); Uzbekistan: Aral Sea, Syr-Dar'inskaya Distr., 30.V.1928, N. Olenev,
V. Popov (11 $\sigma^{7} \sigma^{7}$, ZISP); Golodnaya step' Station [= Gulistan $\left.\left(40.500^{\circ} \mathrm{N} 68.784^{\circ} \mathrm{E}\right)\right]$, Khodzh. U.[=Khodzhentskiy uezd], 17.V.1903, G. Yakobson (1 $\bigcirc^{7}$, ZISP); Kzyl-Grad, Kungrad env.

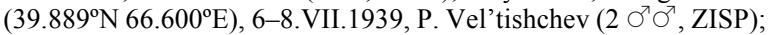
Mekhnatabadskiy Distr., Yangier $\left(40.276^{\circ} \mathrm{N} 68.816^{\circ} \mathrm{E}\right), 15$. V.1980, M. Krivosheina (1 $\sigma^{7}$, ZMUM).

DESCRIPTION. Head grey pollinose except metallic shining frontal vitta and upper face, with bluishgreenish tinge; fronto-orbital plate grey pollinose. 3-4 long fronto-orbital setae, anterior interfrontal setae thin and short, no more than $1 / 3$ of ocellar seta. Postocular setae short and thin. Palpus yellow. Antenna blackish, greyish dusted; arista pectinate on dorsal surface. Gena and parafacial golden-grey dusted.

Thorax black, densely golden-grey pollinose. Scutum subshining with brownish stripes. Acrostichals setulose in many irregular rows, intra-alars absent.

Legs yellow, tarsi of all legs sometimes darkened dorsally.

Abdomen black, grey pollinose, subshining with bluish-green tinge. Tergite 5 about 2-2.5 times as long as tergite 4. Male genitalia large, as long as tergites $4+5$. Epandrium round-oval in dorsal (posterior) view, surstyli thin and curved, parallel-sided apically (Fig. 25). Epandrium parallel-sided in lateral view (Fig. 34); surstylus straight, narrowed apically, with small median emargination. Aedeagus with broad tip in lateral view (Fig. 7). Gonite stout at base with slender tip in lateral view (Fig. 16).

NOTE. The species resembles E. attica in yellow coloration of legs; it is the only species with thin and curved surstyli in dorsal view; this character is clearly 

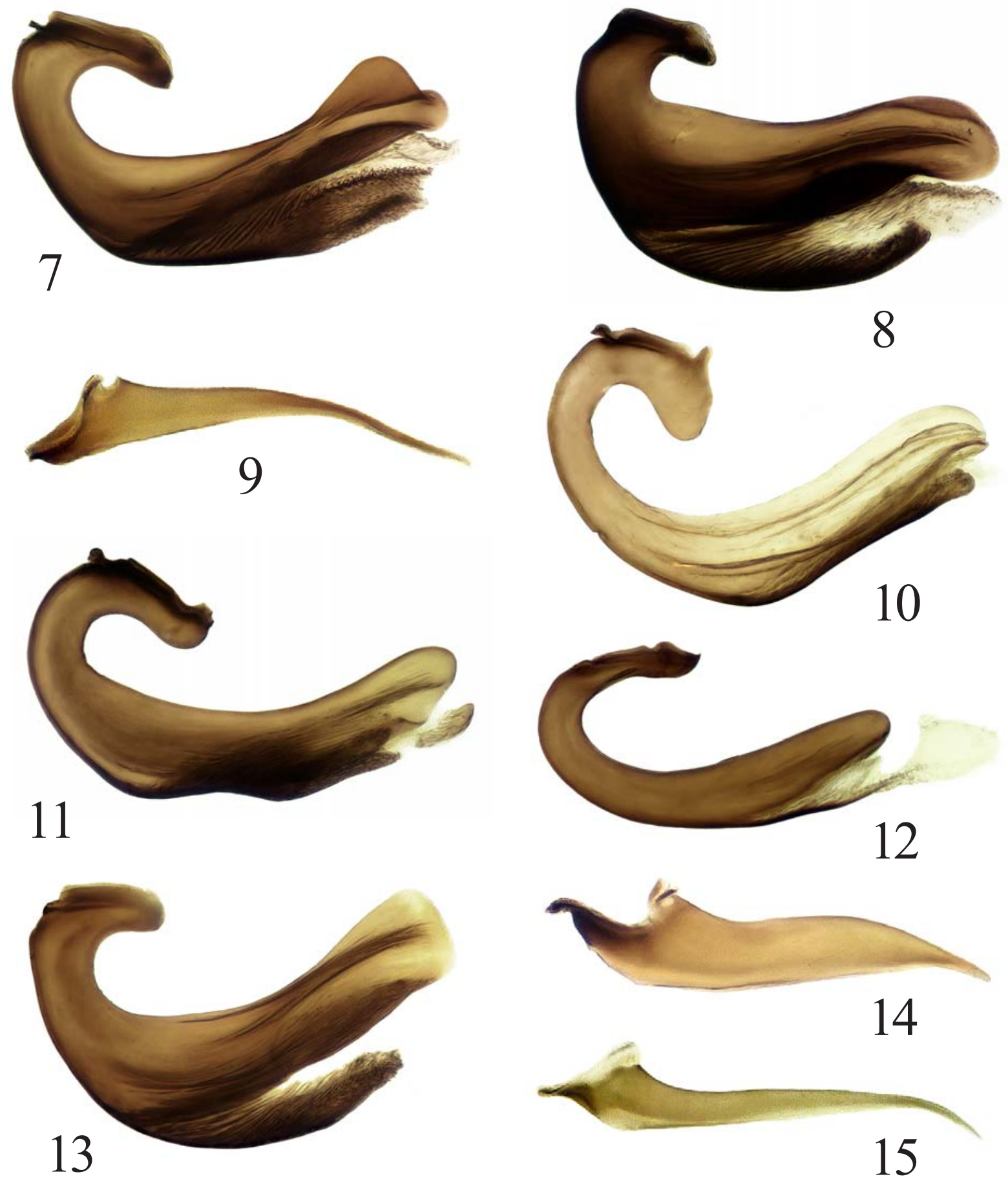

Figs 7-15. Aedeagus of Ephydra spp., lateral view: $7-$ E. afghanica Dahl; $8-$ E. attica Becker; $9-$ E. glauca Meigen; $10-E$. japonica Miyagi; $11-$ E. macellaria Egger; $12-$ E. pseudomurina Krivosheina; $13-$ E. riparia Fallén; $14-$ E. sholtzi Becker; $15-$ E. villosa sp.n.

Рис. 7-15. Эдеагус Ephydra spp., сбоку: $7-$ E. afghanica Dahl; $8-$ E. attica Becker; 9-E. glauca Meigen; $10-$ E. japonica Miyagi; $11-$ E. macellaria Egger; $12-$ E. pseudomurina Krivosheina; $13-$ E. riparia Fallén; $14-E$. sholtzi Becker; $15-E$. villosa sp.n.

visible even in dry specimens.

DISRTIBUTION. Russia: Astrakhan (incl. Krivosheina [2010]), Khakassia. - Palearctic: Afghanistan, Azerbaijan, Bulgaria, China, Jordan, Mongolia,
Turkey, Turkmenistan, Uzbekistan (incl. Wirth [1975]; Krivosheina [1986]; Mathis \& Zatwarnicki [1995]; Hu \& Yang [2002]; Beschovski [2009]); Armenia (first record), Kyrgyzstan (first record). 


\section{Ephydra attica Becker, 1896}

Figs 8, 17, 26, 35 .

attica Becker, 1896: 222 (Ephydra). Type-locality: Athens (Greece).

MATERIAL. Astrakhan' Oblast: Lake Baskunchak $\left(48.193^{\circ} \mathrm{N}\right.$

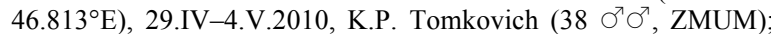
same place, 17.VI.2003, A. Ovchinnikov (3 $\sigma^{7} \sigma^{7}$, ZISP); Kharabali $\left(47.393^{\circ} \mathrm{N}, 47.248^{\circ} \mathrm{E}\right)$, salt lake, 4.VI.2009, P. Mel'nik (6 $\sigma^{7} \sigma^{7}$, ZMUM); Crimea: Kerch env. $\left(45.2^{\circ} \mathrm{N} 36.1^{\circ} \mathrm{E}\right), 26 . I V .2014, \mathrm{~N}$ Vikhrev (1 $\sigma^{7}$, ZMUM); North Ossetia - Alania: Sukhotskoe $\left(43.674^{\circ} \mathrm{N} 44.442^{\circ} \mathrm{E}\right), 30 \mathrm{~km} \mathrm{SW}$ of Mozdok, 3.VIII.1988, A. Ozerov (5 $\sigma^{7} \sigma^{7}$, ZMUM); Stavropol' Krai: Stavropol' $\left(45.045^{\circ} \mathrm{N}\right.$ 41.973E), 5.V.1909, B. Uvarov (1 $\sigma^{\gamma}$, ZISP).

ADDITIONAL MATERIAL. Azerbaijan: Alekseevka [now Dashtatyuk $\left(38.686^{\circ} \mathrm{N} 48.748^{\circ} \mathrm{E}\right), 12 \mathrm{~km} \mathrm{SW}$ of Lankaran, 25.VIII.1932, D. Znoyko (1 $\sigma^{7}$, ZISP); Kazakhstan: Almaty env. $\left(43.283^{\circ} \mathrm{N} 76.906^{\circ} \mathrm{E}\right), 28 . V I .1944$, A. D'yakonov (1 $\sigma^{7}$, ZISP); Morocco: Qued Tensift $\left(31.726^{\circ} \mathrm{N} 8.326^{\circ} \mathrm{W}\right)$, 21.III. and 25.III.2009, N. Vikhrev (2 $\sigma^{7} \sigma^{7}$, ZMUM); Tadjikistan: Tigrovaya balka Nature Reserve (ca. $37.351^{\circ} \mathrm{N} 68.485^{\circ} \mathrm{E}$ ), Dzhilikul' env., 17.IV.1988, N. Krivosheina (1 $\sigma^{7}$, ZMUM); Turkey: Dilek Milli Park (ca $37.66^{\circ} \mathrm{N} 27.14^{\circ} \mathrm{E}$ ), 12.XII.2006, N. Vikhrev (1 $\sigma^{7}$, ZMUM); Samandag env. $\left(36.089^{\circ} \mathrm{N} 35.975^{\circ} \mathrm{E}\right), 14-16 . I V .2010$, N. Vikhrev (1 $\sigma^{7}$, ZMUM); Kopru $\left(37.075^{\circ} \mathrm{N} 31.232^{\circ} \mathrm{E}\right), 10 . \mathrm{IX} .2009$, N. Vikhrev (1 $\sigma^{7}$, ZMUM); Uzbekistan: Aral Sea, Syr-Dar'inskaya Distr., 30.V.1928, N. Olenev, V. Popov (6 O' $\sigma^{7}$, ZISP).

DESCRIPTION. Head golden or silvery-grey-bluish pollinose except metallic shining frontal vitta and upper face; fronto-orbital plate grey pollinose. 3-4 long fronto-orbital setae, 3 interfrontal setae thin and short, anterior seta strongest, $1 / 3$ as long as ocellar. Postocular setae short and thin. Palpus yellow. Antenna blackish, greyish dusted; arista pectinate on dorsal surface on $2 / 3$. Gena and parafacial grey dusted.

Thorax black, grey pollinose. Scutum grey pollinose, subshining with greenish tinge. Acrostichals setulose in many irregular rows, intra-alars absent.

Legs. Femora from yellow to black, grey dusted; tibiae and tarsi yellow, darkened dorsally.

Abdomen black, grey pollinose, subshining with bluish tinge. Tergite 5 about to $1.5-2$ times as long as tergite 4. Male genitalia large, as long as tergites $4+5$. Epandrium round-oval in dorsal (posterior) view (Fig. 26), a little wider near base of surstyli; surstyli straight and widened at apex, parallel-sided apically, with many relatively long perpendicular hairs. Epandrium parallel-sided in lateral view (Fig. 35); surstylus straight, broad, pointed apically. Aedeagus with round tip in lateral view (Fig. 8). Gonite stout at base with round tip in lateral view, subapical process of moderate length (Fig. 17).

NOTE. The species resembles $E$. afghanica in yellow coloration of legs in some specimens, but differs in the shape of surstyli which are widened apically in dorsal view and covered with setae. The specimens with dark tibiae may be separated from E. macellaria by the presence of long setae on surstyli; this character is clearly visible even in dry specimens.

DISRTIBUTION. Russia: Astrakhan Oblast (incl. Krivosheina [2010]), Crimea (incl. Krivosheina [2014]), North Ossetia - Alania, Orenburg Oblast [Shayhutdinova, Krivosheina, 2020], Stavropol' Krai. — Palearctic: Algeria, Bulgaria, Canary Islands, Greece, Egypt,
Turkmenistan [Wirth, 1975; Mathis \& Zatwarnicki, 1995; El-Hawagry et al., 2018; Krivosheina, 2010]; Azerbaijan (first record), Kazakhstan (first record), Morocco (first record), Tadjikistan (first record), Turkey (first record), Uzbekistan (first record).

\section{Ephydra glauca Meigen, 1830}

Figs 1, 3, 9, 18, 27, 36.

glauca Meigen, 1830: 120 (Ephydra). Type-locality: not given (? Germany).

obscuripes Becker, 1896: 222 (Ephydra). Type-locality: Sarepta [part of Volgograd] (Russia).

MATERIAL. Altai: Klyuchi $\left(52.25^{\circ} \mathrm{N}, 79.16^{\circ} \mathrm{E}\right)$, lake Klyuchevskoe bank, 20.VI.2009, O. Kosterin (1 O, 1 क, ZMUM); Lake Kolyvanovskoe $\left(51.363^{\circ} \mathrm{N} 82.191^{\circ} \mathrm{E}\right)$, 8.IX.2007, O. Kosterin (1 $\sigma^{7}, 1$, ZMUM); Astrakhan' Oblast: salt lake Baskunchak $\left(48.193^{\circ} \mathrm{N} 46.813^{\circ} \mathrm{E}\right)$, near fresh pond, 2-6.V.2010, K. Tomkovich (7 $\sigma^{7} \sigma^{7}, 1$, ZMUM); same place, 17.06.2003, A. Ovchinnikov $\left(6\right.$ ○ $^{7}, 1$ ㅇ, ZISP); Khakassia: Borets $\left(54.445^{\circ} \mathrm{N} 90.33^{\circ} \mathrm{E}\right)$, salt pond, 28.VI.2011, K. Tomkovich (1 +, ZMUM); Lake Shunet $\left(54.419^{\circ} \mathrm{N} 90.227^{\circ} \mathrm{E}\right), 5$.VII.1897, Yu. Vagner (1 O', ZISP); Pri-

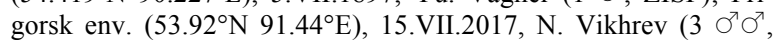
ZMUM); salt lake Beljo (54.65 $\left.{ }^{\circ} \mathrm{N} 90.18^{\circ} \mathrm{E}\right), 382 \mathrm{~m}, 1-3 . \mathrm{VII} .2011$, K.P. Tomkovich (4 $\sigma^{7} \sigma^{7}$, ZISP); Novosibirsk Oblast: Novosibirsk, Zaeltsovsky Park, Ob'-river bank, 15.IX.2007 (2 $\sigma^{7} \sigma^{\top}, 2$, 2 , ZMUM); Novosibirsk, Akademgorodok $\left(54.825^{\circ} \mathrm{N} 83.113^{\circ} \mathrm{E}\right)$, 1.VII.2009, O. Kosterin (3 $\sigma^{7} \sigma^{7}, 1$ †, ZMUM); Omsk Oblast: Cherlak-Krasny Oktyabr' env. (54.133 $\left.{ }^{\circ} \mathrm{N} 75.011^{\circ} \mathrm{E}\right), 30 . V .2009$, O. Kosterin (1 $\sigma^{7}$, ZMUM); Omsk, Solenoe Lake $\left(54.885^{\circ} \mathrm{N}\right.$ 73.347 E), 30.VI.2008, O. Kosterin ( $2 \sigma^{\top} \sigma^{7}, 2$ ○ᄋ, ZMUM); same place, 29.VI.2013, O. Kosterin (2 $\sigma^{7} \sigma^{\top}$, ZMUM); Omsk, River Irtysh left floodland $\left(54.952^{\circ} \mathrm{N} 73.355^{\circ} \mathrm{E}\right)$, reeds and salinas, 3.IV.2008, O. Kosterin (4 $\sigma^{\top} \sigma^{\top}, 3$ 9, , ZMUM); Orenburg Oblast: Irgizly $\left(52.959^{\circ} \mathrm{N} 57.024^{\circ} \mathrm{E}\right), 24 . \mathrm{VI} .1899$, Yakobson \& Shmidt (2 $\sigma^{7}$, ZISP); Stavropol' Krai: Bol'shoy Tambukan (ca. $43.961^{\circ} \mathrm{N}$ $\left.43.158^{\circ} \mathrm{E}\right), 1909$, A. Skorikov, "Ephydra obscuripes Beck., Stack-

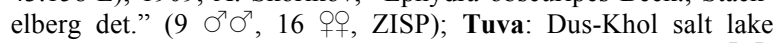
$\left(51.36^{\circ} \mathrm{N} 94.45^{\circ} \mathrm{E}\right), 700 \mathrm{~m}, 2-5$.VII.2017, N. Vikhrev $\left(2 \Im^{7} \sigma^{7}\right.$, ZMUM); Yakutia: Lake Abalakh $\left(61.676^{\circ} \mathrm{N} 131.178^{\circ} \mathrm{E}\right), 6 \mathrm{~km} \mathrm{~W}$ of Ele-chey, saline meadow near lake, 16.VIII.2011, L. Sivtseva (1 $\sigma^{7}$, ZMUM); Lake Mokhsogollokh (salt), Kempendyay env. $\left(62.033^{\circ} \mathrm{N} 118.651^{\circ} \mathrm{E}\right)$, on water surface, 23.VI.2011, L. Sivtseva (3 $\bigcirc^{7}, 1$, ZMUM); Lake Muosany, $52 \mathrm{~km}$ ENE of Kempendyay $\left(62.278^{\circ} \mathrm{N} 119.44^{\circ} \mathrm{E}\right)$, on water surface, 23.VI.2011, L. Sivtseva (1 $\mathrm{O}^{\top}, 6$ 우, ZMUM); Zabaikalsky Krai, $20 \mathrm{~km}$ NE of Solov'evsk

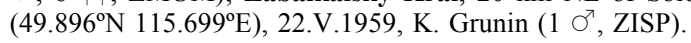

ADDITIONAL MATERIAL. Kazakhstan: SW Kazakhstan, Narynkol [= Raiymbek] District, lake Tuzkol $\left(43.006^{\circ} \mathrm{N} 79.971^{\circ} \mathrm{E}\right)$, 31.VIII.1959, P. Lehr (3 $\sigma^{7} \sigma^{7}$, ZISP); Shortandy $\left(51.698^{\circ} \mathrm{N}\right.$ $\left.70.997^{\circ} \mathrm{E}\right)$, 19.VI.1938, B. Kuzin (1 O', ZISP); May-Emer, Turgaysk. O., 29.VI.1928, V. Popov (1 $\sigma^{\top}, 1$ ㅇ, ZISP); Borovoe [Burabay, $\left.53.083^{\circ} \mathrm{N} 70.307^{\circ} \mathrm{E}\right), 2$, 12.VII., 14.VII.2006, N. Dvoretskaya (4 90, ZMUM); $36 \mathrm{~km} \mathrm{SSE}$ of Aktogay $\left(46.64^{\circ} \mathrm{N} 79.861^{\circ} \mathrm{E}\right), 400$ m, 13.VI.2014, V. Zinchenko (1 $\sigma^{7}$, ZISP); Ukraine: Golaya Pristan' $\left(46.524^{\circ} \mathrm{N} 32.521^{\circ} \mathrm{E}\right), 18$.VII.1977, on decaying plants of salt lake, E. Klechkovskiy ( $3 \sigma^{\top} \sigma^{7}$, ZMUM); Chernomorskiy Nature Reserve (ca. $46.314^{\circ} \mathrm{N} 31.890^{\circ} \mathrm{E}$ ), cordon Geroyskiy, 6.VII.1977, E. Klechkovskiy ( 2 ㅇ, ZMUM); Aleshki $\left(46.62^{\circ} \mathrm{N} 32.719^{\circ} \mathrm{E}\right)$, 18.VI.1926, L. Zimin (1 $\sigma^{7}$, ZISP); Uzbekistan: Aral Sea, SyrDar'inskaya Distr., 30.V.1928, N. Olenev, V. Popov (5 $\sigma^{\top} \sigma^{\top}, 1 \sigma^{\top}$, ZISP); Ayakguzhumdy (40.738 $\left.{ }^{\circ} \mathrm{N} 63.745^{\circ} \mathrm{E}\right), 7 . V I .1965$, E. Nartshuk (1 $\sigma^{\prime}$, ZISP).

DESCRIPTION. Body-length 4.5-5.5 mm.

Head (Fig. 1) grey pollinose except metallic shining frontal vitta, with greenish tinge; upper face pollinose; fronto-orbital plate grey or brownish pollinose. 3-4 (rarely 5) long fronto-orbital setae, 3 pairs of interfrontal setae, of which anterior strong, 1/2 as long as 

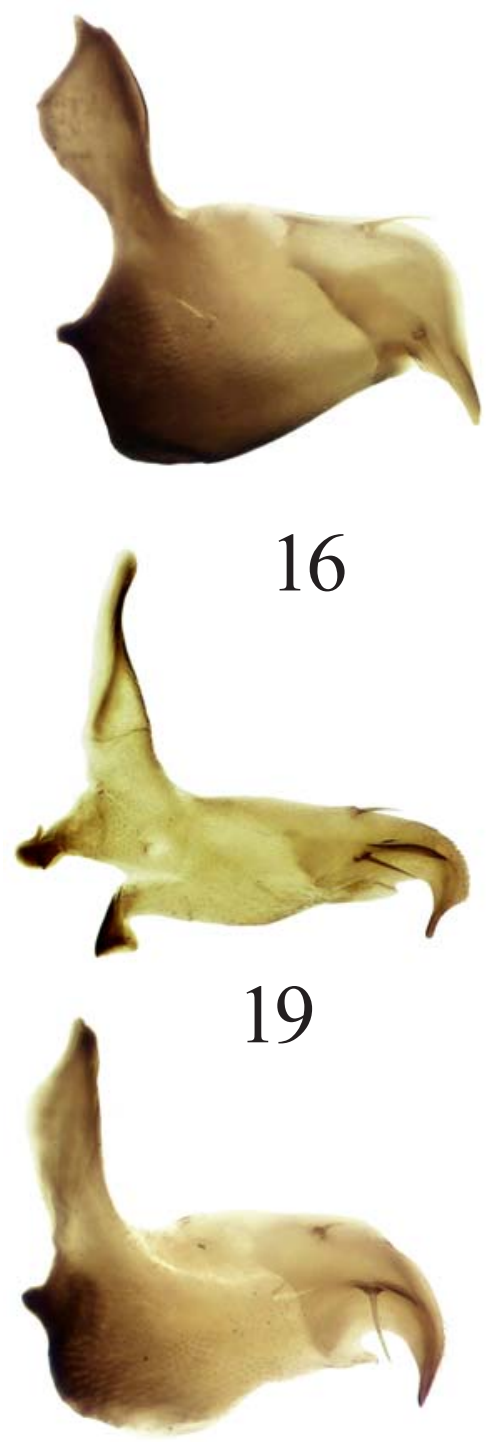

22

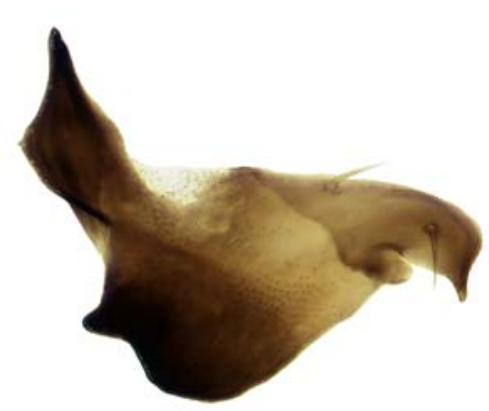

17

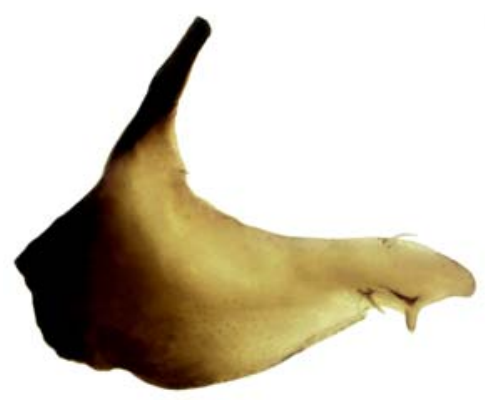

20

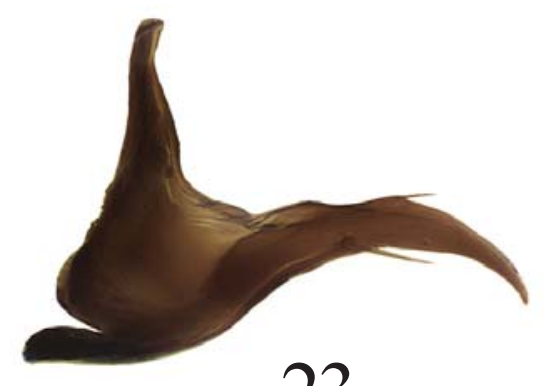

23

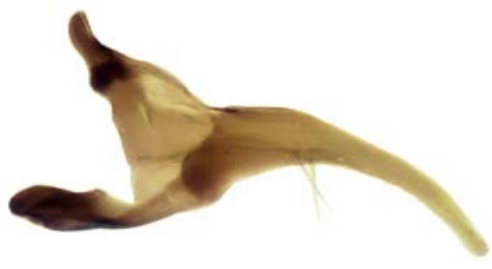

18

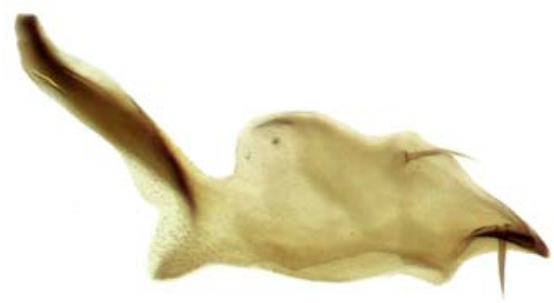

21

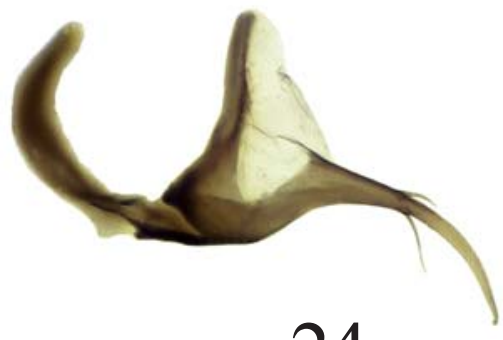

24

Figs 16-24. Gonite of Ephydra spp., lateral view: 16-E. afghanica Dahl; $17-$ E. attica Becker; $18-$ E. glauca Meigen; $19-E$. japonica Miyagi; $20-$ E. macellaria Egger; $21-$ E. pseudomurina Krivosheina; 22 - E. riparia Fallén; 23 - E. sholtzi Becker; $24-$ E. villosa sp.n.

Рис. 16-24. Гонит Ephydra spp., сбоку: 16 - E. afghanica Dahl; $17-$ E. attica Becker; $18-$ E. glauca Meigen; $19-$ E. japonica Miyagi; $20-$ E. macellaria Egger; $21-$ E. pseudomurina Krivosheina; $22-$ E. riparia Fallén; $23-$ E. sholtzi Becker; $24-E$. villosa sp.n.

ocellar. Postocular setae well developed. Palpus dark, grey pollinose. Antenna blackish, greyish dusted; arista short pubescent on dorsal surface on basal $1 / 2$. Gena and parafacial greyish pollinose.

Thorax black, densely grey pollinose, not subshining. Scutum with brownish fuse spots and stripes (Fig. $3)$. Acrostichals setulose in two rows, intra-alars $1+(0$ 2) (Fig. 3).

Legs black, densely grey dusted; tarsi of all legs yellowish ventrally.

Abdomen black with greenish tinge, densely grey pollinose. Tergite 5 approximately equal in length to tergite 4. Male genitalia small, as long as tergite 5 . Epandrium elongate in dorsal (posterior) view (Fig. 27), wider in cercal half; surstylus narrowed apically. Epandrium parallel-sided in lateral view (Fig. 36); surstylus is at obtuse angle to epandrium, bifurcate apically, one lobe thin. Aedeagus with slender pointed tip in lateral view (Fig. 9). Gonite stout at base with curved distal part and slender tip in lateral view (Fig. 18).

NOTE. The species differs in acrostichal setae arranged in two rows, presence of well developed postocular setae and dense grey pollinosity, upper face, scutum and abdomen not subshining. 
DISTRIBUTION. Russia: Altai, Astrakhan' Oblast, Khakassia, Novosibirsk Oblast, Omsk Oblast, Orenburg Oblast, Stavropol' Krai, Tuva, Volgograd Oblast, Yakutia, Zabaikalsky Krai. - Palearctic: Algeria, Bulgaria, France, Hungary, Italy, Mongolia, Poland, Romania, Turkey [Mathis, Zatwarnicki, 1995]. Kazakhstan (first record), Ukraine (first record), Uzbekistan (first record).

\section{Ephydra japonica Miyagi, 1966} Figs 10, 19, 28, 37.

japonica Miyagi 1966: 137 (Ephydra). Type-locality: Hokkaido: Sapporo (Japan).

MATERIAL. Primorsky Krai: Kedrovaya Pad' Nature Reserve $\left(43.104^{\circ} \mathrm{N} 131.512^{\circ} \mathrm{E}\right), 26$. VI.1940, A. Monchadskiy (1 $\sigma^{7}$, ZISP); same place, 25.VIII.1980, A. Shatalkin (2 $\sigma^{7} \sigma^{7}$, ZMUM); $40 \mathrm{~km}$ SO of Ussuriysk (43.634 $\left.{ }^{\circ} \mathrm{N} 132.222^{\circ} \mathrm{E}\right), 30$.VII.1983, 6.VIII 1983, A. Ozerov (2 ○ $\sigma^{7}$, ZMUM); Melkovodnoe $\left(42.861^{\circ} \mathrm{N} 133\right.$. $\left.618^{\circ} \mathrm{E}\right)$, A. Ozerov (9 $\left.\sigma^{\top} \sigma^{\top}, \mathrm{ZMUM}\right)$; Posiet $\left(42.67^{\circ} \mathrm{N} 130.82^{\circ} \mathrm{E}\right)$ seashore, 25-30.VI. and 31.VII.2014, N. Vikhrev (3 $\sigma^{\top} \sigma^{7}$, ZMUM); Andreevka $\left(42.7^{\circ} \mathrm{N} 131.1^{\circ} \mathrm{E}\right), 25-30 . V I .2014$, N. Vikhrev (1 $\sigma^{\prime \prime}$, ZMUM).

DESCRIPTION. Head golden-grey or bluish-grey pollinose except metallic shining frontal vitta and upper face, with bluish-greenish tinge; fronto-orbital plate grey or brownish pollinose. 3-4 long fronto-orbital setae, anterior interfrontal setae thin and short, no more than $1 / 3$ of ocellar. Postocular setae thin and short. Palpus yellow basally and darkened apically. Antenna blackish, brownish dusted; arista short pubescent on dorsal surface on basal $1 / 2$ or a little more. Gena grey dusted, parafacial golden.

Thorax black, densely brown pollinose. Scutum dark subshining with brownish stripes. Acrostichals setulose in many irregular rows, intra-alars absent.

Legs. Femora black or yellowish, grey dusted, tibia and tarsi of all legs yellowish, darkened dorsally.

Abdomen black, grey pollinose, subshining with greenish tinge. Tergite 5 golden in some specimens, 2 times as long as tergite 4 . Male genitalia large, as long as tergites $4+5$. Epandrium round-oval in dorsal (posterior) view (Fig. 28), surstyli straight and not widened at apex, parallel apically. Epandrium of one width in lateral view (Fig. 37); surstylus broad, narrowed but not pointed, round and curved apically. Aedeagus with round tip in lateral view (Fig. 10). Gonite with curved narrow tip in lateral view (Fig. 19).

NOTE. The species is close to E. riparia, differing from it in the shape of surstyli, which are not widened apically in dorsal view, and in rounded apex of surstyli in lateral view. Besides, the abdominal tergite 5 of male is golden, contrasting to grey-greenish coloration of other tergites. Our figures of surstyli are made from strictly dorsal position. Turned a little laterally one can see position given by Wirth [1975].

DISRTIBUTION. Russia: Primorsky Krai. - Palearctic: China, Japan (Hokkaido), Korea (South); Oriental: Japan (Ryukyu Islands, Bonin Islands) [Wirth, 1975].

\section{Ephydra macellaria Egger, 1862}

Figs 11, 20, 29, 38. (Italy).

macellaria Egger 1862: 779 (Ephydra). Type-locality: Trieste alandica Frey 1909: 12 (Ephydra). Type locality: Åland Islands, Hammarudda (Finland).

MATERIAL. Altai: Kosh-Agach env. $\left(50.01^{\circ} \mathrm{N} 88.66^{\circ} \mathrm{E}\right), 1750$ m, 2-4.VII.2016, N. Vikhrev (3 $\sigma^{7} \sigma^{7}$, ZMUM); Karelia: Primorsky env. $\left(66.549^{\circ} \mathrm{N}, 33.133^{\circ} \mathrm{E}\right)$, salt pool, 1.VIII.2018, A.V. Markov (2 $0^{7} O^{7}$, ZMUM).

ADDITIONAL MATERIAL. Kyrgyzstan: Choktal env. $\left(42.58^{\circ} \mathrm{N} 76.75^{\circ} \mathrm{E}\right), 1600 \mathrm{~m}, 19-22 . I X .2013, \mathrm{~N}$. Vikhrev (1 $\sigma^{7}$, ZMUM); Mongolia: Uver-Khangayskiy aymak, Samon Boyan Ulan, $1800 \mathrm{~m}, 3$. VI.1987, O. Gorbunov (2 Ơ $\sigma^{7}$, ZMUM); Tadjikistan: Tigrovaya balka Nature Reserve, 15.IV.1986, N. Krivosheina (1 $\mathrm{O}^{7}$, ZMUM); Lochor $\left(38.383^{\circ} \mathrm{N} 68.704^{\circ} \mathrm{E}\right), 19 . \mathrm{V} .1939$, E. Smirnov ( $1 \mathrm{O}^{7}$, ZMUM); Turkmenistan: Kushka env. (35.281 $\left.\mathrm{N} 62.342^{\circ} \mathrm{E}\right)$, 20.V.1991, A. Ozerov (1 $\sigma^{7}$, ZMUM); $30 \mathrm{~km} \mathrm{NW}$ of Ashgabat $\left(38.164^{\circ} \mathrm{N} 57.960^{\circ} \mathrm{E}\right), 16 . I V .1983,18 . I V .1983$, M. Krivosheina (2 $\mathrm{O}^{7} \mathrm{O}^{\top}$, ZMUM); Ashgabat $\left(37.934^{\circ} \mathrm{N} 58.387^{\circ} \mathrm{E}\right), 15 . X I .1983, \mathrm{~N}$. Krivosheina (2 $\sigma^{7} \sigma^{\top}$, ZMUM); Morocco: $\mathrm{S}$ of Essaouira, $31.37^{\circ} \mathrm{N}$, $9.71^{\circ} \mathrm{W}$, 25.III.2009, pond, 27.III.2009, sand dune, 28.III.2009, N. Vikhrev (6 $0^{7} \sigma^{7}$, ZMUM); Marrakech, Qued Tensift, 21.III.2009 N. Vikhrev (1 $0^{7}$, ZMUM); Canary Islands: Tenerife, Teno Peninsula, temporary pool, lowland, 25-30.III.2011, N. Vikhrev (10 $\sigma^{7} \sigma^{7}$, ZMUM).

DESCRIPTION. Head whitish-grey (alandica) or grey (macellaria) pollinose except metallic shining frontal vitta and upper face, with bluish-greenish tinge; fronto-orbital plate grey pollinose. 3 long fronto-orbital setae, anterior interfrontal thin and about $1 / 3$ of ocellar. Postocular setae thin and short. Palpus yellowgrey to dark grey. Antenna black, brown dusted; arista pectinate on dorsal surface. Gena and parafacial whitish-grey or greyish-golden dusted.

Thorax black, densely whitish-grey or grey pollinose. Scutum whitish-grey pollinose or metallic subshining. Acrostichals setulose in many irregular rows, intra-alars absent.

Legs black, densely greyish pollinose; tarsi yellowish.

Abdomen black, densely whitish or grey pollinose, subshining with greenish tinge or without metallic tinge. Tergite 5 about $1.3-1.5$ as long as tergite 4 . Male genitalia large, as long as tergites $4+5$. Epandrium elongate-oval in dorsal (posterior) view (Fig. 29), surstylus narrow and straight, covered with few short hairs. Epandrium parallel-sided in lateral view (Fig. 38), surstyli broad, narrowing and round apically. Aedeagus with round pointed tip in lateral view (Fig. 11). Gonite stout at base with rounded distal part in lateral view, appendage of gonite is located at significant distance from the tip (Fig. 20).

NOTE. The species differs from E. afghanica in dark coloration of fore femora. The absence of long hairs on surtylus and unique shape of gonite with protruding apical lobe and narrow appendage placed at significant distance from it separate E. macellaria from E. attica and make it possible to determine this species with confidence. Mathis \& Zatwarnicki [1995] mentioned "Caucasus" as the area of distribution of this species. However, all specimens from Caucasus, which we found in the collection, belong to E. attica. 


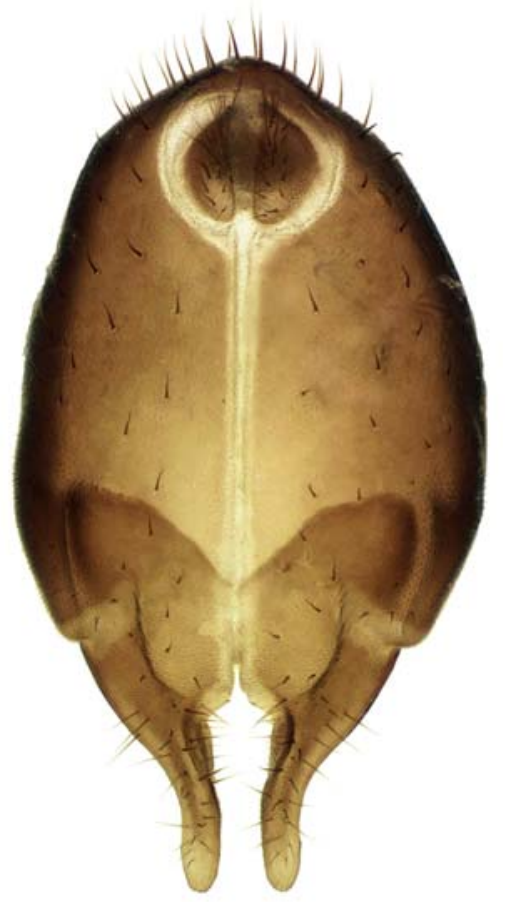

25

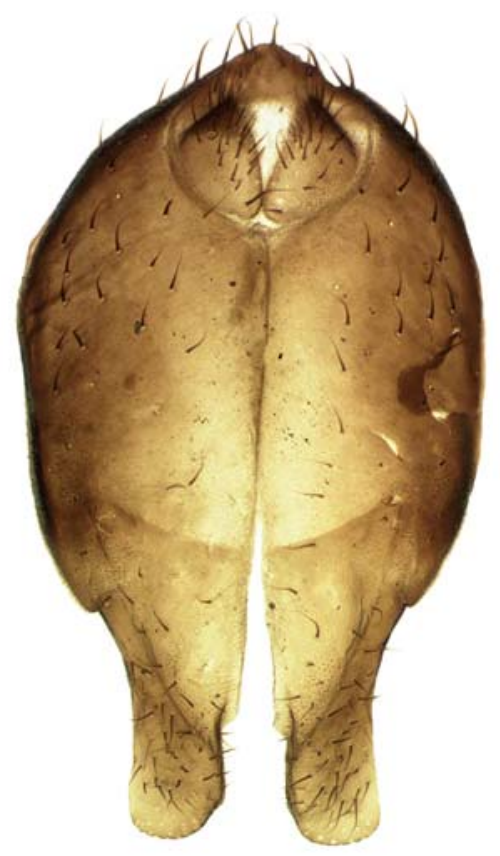

28

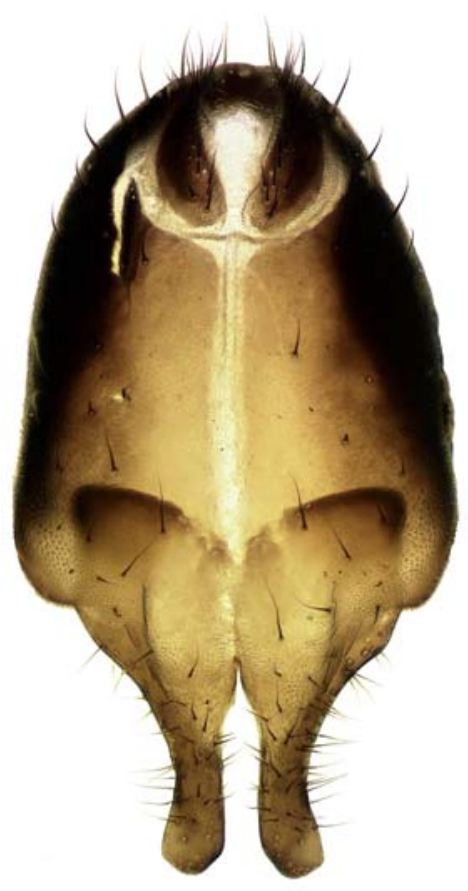

26

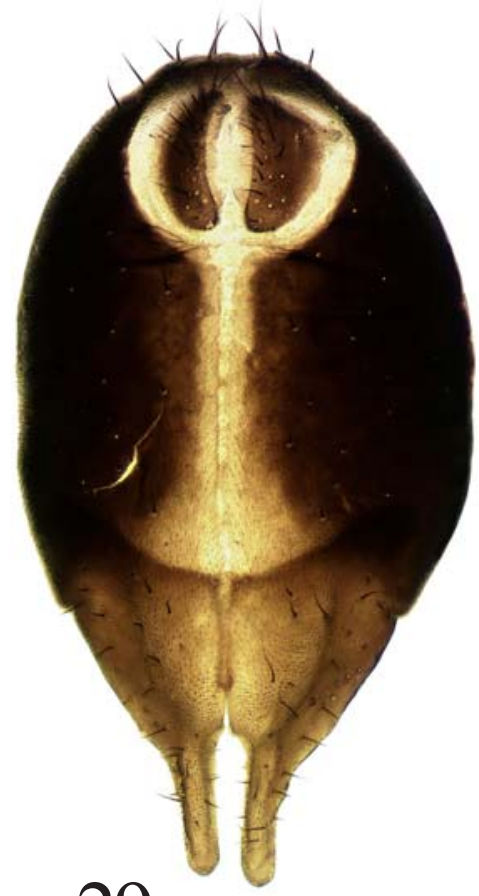

29
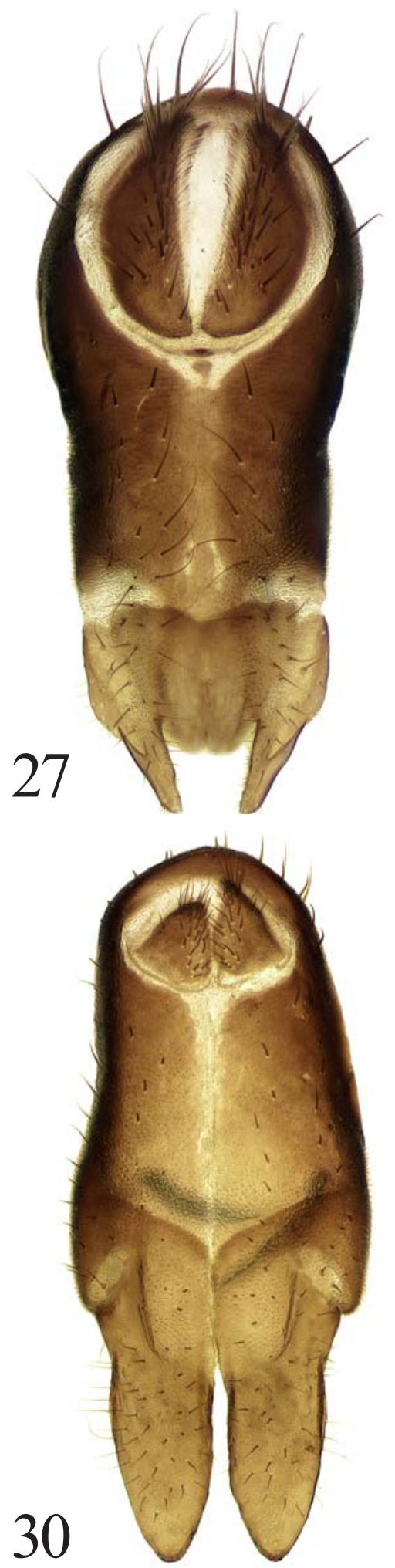

Figs 25-30. Epandrium and surstyli of Ephydra spp., dorsal view: $25-$ E. afghanica Dahl; $26-$ E. attica Becker; $27-$ E. glauca Meigen; $28-$ E. japonica Miyagi; $29-$ E. macellaria Egger; $30-$ E. pseudomurina Krivosheina.

Рис. 25-30. Эпандриум и сурстили Ephydra spp., сверху: 25 - E. afghanica Dahl; 26 - E. attica Becker; 27 - E. glauca Meigen; 28 - E. japonica Miyagi; $29-$ E. macellaria Egger; $30-$ E. pseudomurina Krivosheina. 

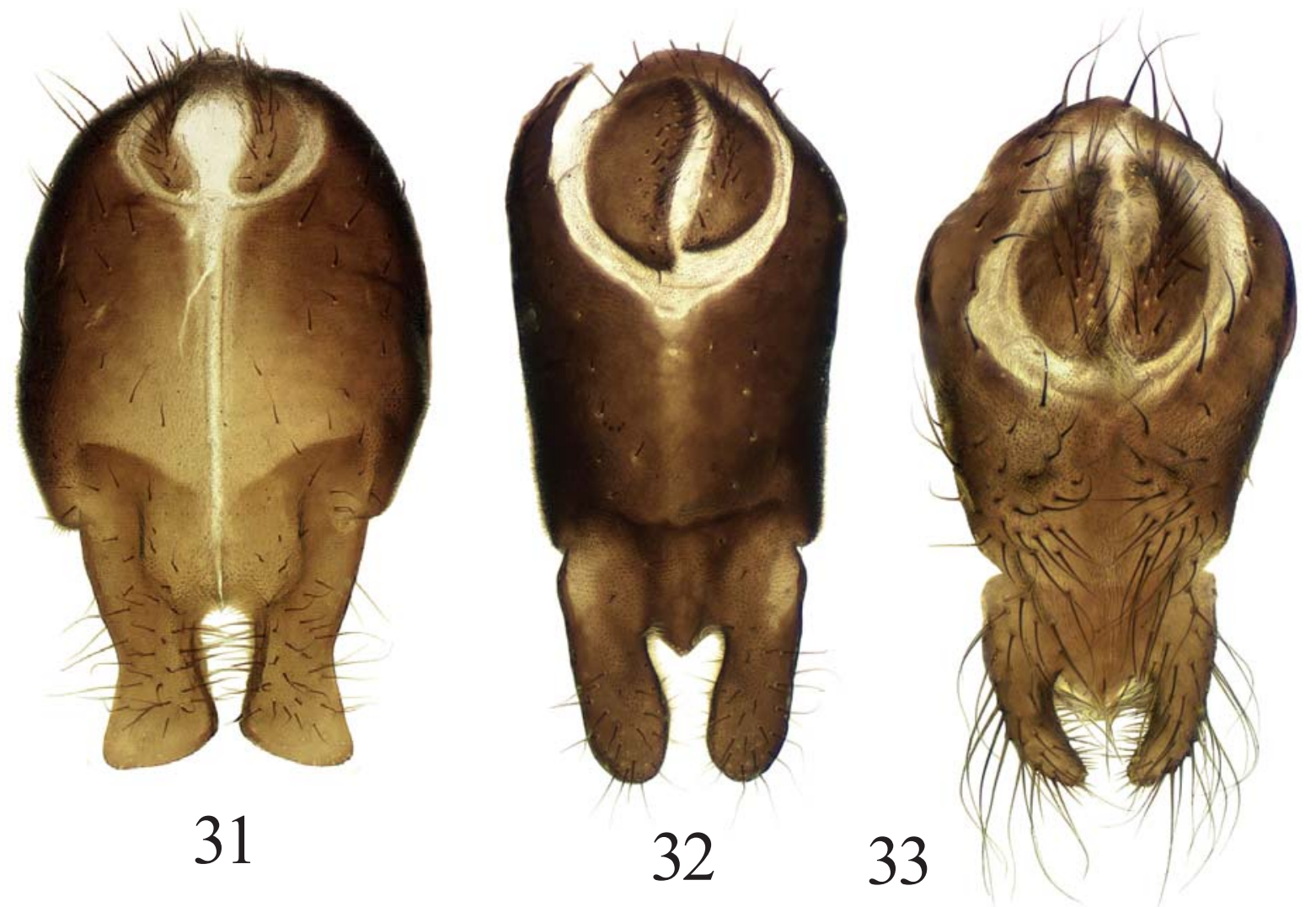

Figs 31-33. Epandrium and surstyli of Ephydra spp., dorsal view: $31-$ E. riparia Fallén; $32-E$. sholtzi Becker; $33-E$. villosa sp.n. Рис. 31-33. Эпандриум и сурстили Ephydra spp., сверху: $31-$ E. riparia Fallén; $32-$ E. sholtzi Becker; $33-E$. villosa sp.n.

The species is divided into 2 subspecies. E. macellaria alandica differs in entirely whitish-grey dusted coloration and is distributed in Finland, Norway and Sweden on the shore of Baltic Sea [Wirth, 1975] and in Russia on the shore of White Sea. The second subspecies E. macellaria macellaria differs in metallic greenish subshining frons, scutum and abdomen and is widely distributed in the Palaearctic and Afrotropical Regions. Male genitalia of these subspecies are identical.

DISTRIBUTION. Russia: Altai, Karelia. - Afrotro-pical: Cape Verde Islands; Palearctic: Afghanistan, Algeria, Azores, Bulgaria, Canary Islands, China (NE Tibet), Cyprus, Czech Republic, Egypt, Finland, France, Germany, Great Britain, Greece, Iran, Italy, Libya, Madeira Islands, Malta, Morocco, Netherlands, Norway, Romania, Sweden, Tunisia, Turkmenistan, Ukraine [Mathis, Zatwarnicki, 1995]; Kyrgyzstan (first record), Mongolia (first record), Tadjikistan (first record).

\section{Ephydra pseudomurina Krivosheina, 1983}

Figs 6, 12, 21, 30, 39.

pseudomurina Krivosheina 1983: 368 (Ephydra). Type-locality: Mekhnatabadskiy Distr., Yangier [40.276 $\left.{ }^{\circ} \mathrm{N} 68.816^{\circ} \mathrm{E}\right](\mathrm{Uz}-$ bekistan).

MATERIAL. Astrakhan' Oblast: salt lake Baskunchak $\left(48.193^{\circ} \mathrm{N} 46.813^{\circ} \mathrm{E}\right), 17.06 .2003$, A. Ovchinnikov (9 $\sigma^{\top} \sigma^{\top}$, ZISP); same place, 2-4.V.2010, K. Tomkovich (3 $\sigma^{7} \sigma^{7}$, ZMUM); Kharabali $\left(47.393^{\circ} \mathrm{N}, 47.248^{\circ} \mathrm{E}\right)$, salt lake, 4.VI.2009, P. Mel'nik (1 $\sigma^{7}$, ZMUM); Crimea: Kerch env. $\left(45.2^{\circ} \mathrm{N} 36.1^{\circ} \mathrm{E}\right), 26 . I V .2014$, N.

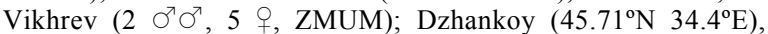
19.V.1963, K. Gorodkov (1 $\sigma^{7}$, ZISP); Koktybel' $\left(44.964^{\circ} \mathrm{N}\right.$ $\left.35.241^{\circ} \mathrm{E}\right), 1-8 . I X .2007, \mathrm{~K}$. Tomkovich (1 ऽ', ZMUM); Lake Koyanskoe $\left(45.025^{\circ} \mathrm{N} 36.111^{\circ} \mathrm{E}\right)$, reared, 2008, A. Przhiboro (1 $\sigma^{\top}$, ZISP); Krasnodar Krai: Ubinskaya $\left(44.74^{\circ} \mathrm{N} 38.54^{\circ} \mathrm{E}\right), 8$. VI.1971, V. Kovalev (1 $\sigma^{7}$, ZMUM); Rostov Oblast: Gigant vill. $\left(46.507^{\circ} \mathrm{N}\right.$ $41.34^{\circ} \mathrm{E}$ ), on the light, 17-22.VI.2009, A. Koval (1 $\sigma^{7}$, ZMUM).

ADDITIONAL MATERIAL. Azerbaijan: Avrora [=Hirkan, $\left.38.67^{\circ} \mathrm{N} 48.799^{\circ} \mathrm{E}\right]$, 19.V.1981, N. Krivosheina (1 ${ }^{7}$, ZMUM); Georgia: Tiflis [=Tbilisi, $41.71^{\circ} \mathrm{N} 44.791^{\circ} \mathrm{E}$ ], 1903, K. Satunin (2 $\bigcirc^{7} \bigcirc^{7}$, ZISP); Kazakhstan: Golodnaya step', 7.VII.1959, P. Lehr (1 $\sigma^{7}$, ZISP); Tadjikistan: Nature Reserve Tigrovaya balka, 15.IV. 1986, 15-17.V.1987, N. Krivosheina (2 $\sigma^{7} \sigma^{7}$, ZMUM); Turkmenistan: Ashgabat $\left(37.934^{\circ} \mathrm{N} 58.387^{\circ} \mathrm{E}\right), 15 . \mathrm{XI} .1983$, N. Krivosheina

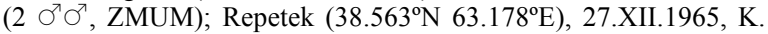
Gorodkov (2 $\sigma^{7} \sigma^{7}$, ZISP); same place, 25.I.1966, V. Zaitzev (1 $\sigma^{7}$, ZISP); Lake Sarygamysh (ca. 42.052 ${ }^{\circ}$ N 57.697E), 19-26.IV.1984, M. Krivosheina (1 $\sigma^{7}$, ZMUM); Ukraine: Odessa, Kuyal'nitskiy liman (ca. $46.565^{\circ} \mathrm{N} 30.717^{\circ} \mathrm{E}$ ), 16.IX.1938, B. Rohdendorf (7 $\sigma^{\top} \sigma^{\top}$, ZMUM); same place, 21.VI.1926, L. Zimin (2 $\sigma^{\top} \sigma^{\top}$, ZISP); Odessa env. (ca. $46.466^{\circ} \mathrm{N} 30.719^{\circ} \mathrm{E}$ ), salt puddle near oil tank, 8.V.1926, L. Zimin (2 O $^{\top} \bigcirc^{7}$, ZMUM); Aleshki $\left(46.62^{\circ} \mathrm{N} 32.719^{\circ} \mathrm{E}\right)$, 18.VI.1926, L. Zimin (2 $\sigma^{7} \sigma^{7}$, ZISP); Chernomorskiy Nature Reserve (ca. $46.314^{\circ} \mathrm{N} 31.890^{\circ} \mathrm{E}$ ), cordon Geroyskiy, 6-7.VII.1977, E. Klechkovskiy (16 $\sigma^{7} \bigcirc^{7}$, ZMUM); Uzbekistan: Mekhnatabadskiy Distr., Yangier $\left(40.276^{\circ} \mathrm{N} 68.816^{\circ} \mathrm{E}\right), 12 . \mathrm{V} .-10 . \mathrm{VI} .1980, \mathrm{M}$. Krivosheina (17 $\Im^{7} \sigma^{7}, 20$ 古, ZMUM); Khiva $\left(41.394^{\circ} \mathrm{N} 60.381^{\circ} \mathrm{E}\right)$, 22.III.1927, L. Zimin; Aral Sea, Syr-Dar'inskaya Distr., 30.V.1928, N. Olenev, V. Popov (1 ๑, ZISP). 
DESCRIPTION. Head golden-grey pollinose except metallic shining frontal vitta and upper face, with bluish-greenish tinge; fronto-orbital plate golden pollinose. 3-4 long fronto-orbital setae, anterior interfrontal thinner and $1 / 3$ as long as ocellar. Postocular setae weak and short. Palpus yellow. Antenna blackish, greyish dusted; arista pectinate on basal half of dorsal surface (Fig. 6). Gena and parafacial greyish dusted.

Thorax black, grey pollinose. Scutum metallic subshining with greenish tinge. Acrostichals setulose in many irregular rows, intra-alars absent.

Legs. Femora yellow, greyish dusted or yellow; tibiae and tarsi of all legs yellow, darkened dorsally.

Abdomen black, grey pollinose, metallic subshining with greenish tinge. Tergite 5 about 1.5-2.0 times as long as tergite 4. Male genitalia large, as long as tergites $4+5$. Epandrium elongate in dorsal (posterior) view (Fig. 30); surstylus broad, rounded apically. Epandrium parallel-sided in lateral view (Fig. 39); surstylus with oval median protuberance. Aedeagus with rounded tip in lateral view (Fig. 12). Gonite stout at base in lateral view, bifurcate apically, of complex shape, giving different images if slightly rotated (Fig. 21).

NOTE. Wirth [1975] described E. murina from the territories of Iraq (holotype), Bulgaria, Iran, Romania, Turkey and Ukraine. The figures of surstyli dorsally were closer to E. flavipes, relatively broad basally and evenly tapering apically. Krivosheina [1983] described the species E. pseudomurina from Uzbekistan; surstyli of this species are broad almost to the tip and narrowed at the very apex. The same species was described by $\mathrm{Hu}$ \& Yang [2001], who did not see the previous work, under the name of E. hejingensis $\mathrm{Hu} \&$ Yang, 2001. Beschovsky [2009] gave figures of surstyli the same as in E. pseudomurina and determined this species as $E$. murina. Przhiboro \& Shadrin [2012] registered the same species in Russia under the name of E. murina. Krivosheina [2010] obviously had two morphological forms of $E$. pseudomurina. However we discovered specimens of Ephydra from Tajikistan, which surstyli are closer to the figures given for E. murina by Wirth [1975]. This prevented us from synonymizing pseudomurina, hejingensis and murina in the present work. We leave the solution to this question for the future, when the holotype of E. murina becomes available for study.

DISRTIBUTION. Russia: Astrakhan' Oblast (incl. Krivosheina [2010]), Crimea (incl. Przhiboro \& Shadrin, [2012]; Krivosheina [2014]), Krasnodar Krai, Orenburg Oblast [Shayhutdinova, Krivosheina, 2020], Rostov Oblast. - Palearctic: Turkmenistan, Uzbekistan [Krivosheina, 1983, 1986, 2010; Mathis, Zatwarnicki, 1995]; Azerbaijan (first record), Georgia (first record), Kazakhstan (first record), Tadjikistan (first record), Ukraine (first record).

\section{Ephydra riparia Fallén, 1813}

Figs 4, 5, 13, 22, 31, 40.

riparia Fallén, 1813: 246 (Ephydra). Type-locality: Sweden.

albula Meigen, 1830: 115 (Ephydra). Type-locality: not given (? Germany) salina von Heyden, 1843: 228 (Ephydra). Type-locality: Creuznach, Rhein-Preussen (Germany).

halophila von Heyden, 1844: 203 (Coenia). Type-locality: Nauheim (Germany)

salinae Zetterstedt, 1846: 1812 (Ephydra). Type-locality: "Gryphia" [= Pomerania: Gryfice] (Poland).

strenzkei Giordani Soika, 1960: 456 (Ephydra). Type-locality: Wilhelmshaven (Germany).

MATERIAL. Amur Oblast: Zeya $\left(54.087^{\circ} \mathrm{N} 126.871^{\circ} \mathrm{E}\right)$, 20.VI.1978, A. Shatalkin (1 $\sigma^{7}$, ZMUM); Chukotka: Anadyr $\left(64.725^{\circ} \mathrm{N} 177.471^{\circ} \mathrm{E}\right)$, swampy lake shore, 13.VIII.1966, K. Gorodkov (22 $\sigma^{\top} \sigma^{7}, 12$ 우, ZISP); Goryachie klyuchi $\left(54.512^{\circ} \mathrm{N}\right.$ $\left.159.973^{\circ} \mathrm{E}\right)$, on the surface of a puddle, water $\mathrm{t} 37^{\circ}$, air $\mathrm{t} 9^{\circ}$, 7.VIII.1963, K. Gorodkov (23 $\sigma^{7} \sigma^{7}$, ZISP); Crimea: Dzhankoy $\left(45.719^{\circ} \mathrm{N} 34.407^{\circ} \mathrm{E}\right), 19 . V .1963, \mathrm{~K}$. Gorodkov (2 $\sigma^{\top} \sigma^{\top}$, ZISP); Lake Koyanskoe $\left(45.025^{\circ} \mathrm{N} 36.111^{\circ} \mathrm{E}\right)$, reared, 2008, A. Przhiboro (1 $\sigma^{7}$, ZISP); Bashkiria: Muraptal Distr., Kazlair saltish $\left(52.455^{\circ} \mathrm{N}\right.$ $\left.55.76^{\circ} \mathrm{E}\right)$, K. Tomkovich (1 $\sigma^{7}$, ZMUM); Buryatia: Kyakhta $\left(50.353^{\circ} \mathrm{N} 106.448^{\circ} \mathrm{E}\right)$, waterlogged meadow, 25.VIII.1958, K. Gorodkov (1 $\sigma^{7}$, ZISP); $15 \mathrm{~km}$ E of Kyakhta, solonchak, $\left(50.337^{\circ} \mathrm{N}\right.$ 106.700 E), 24.VII.2012, A. Medvedev (4 $\sigma^{7} \sigma^{7}$, ZMUM); Kamchatka Krai: Apuka $\left(60.444^{\circ} \mathrm{N} 169.601^{\circ} \mathrm{E}\right)$, an island at the mouth of the Apuka River, on rotten puddles, 7.VIII.1959, K. Gorodkov (14 $\bigcirc^{7} \sigma^{7}$, ZISP); Na-lychevo $\left(53.507^{\circ} \mathrm{N} 158.756^{\circ} \mathrm{E}\right), 2 . X .2009$, L. Lobkova (1 $0^{7}$, ZMUM); Petropavlovsk-Kamchatsky $\left(53.013^{\circ} \mathrm{N}\right.$ $\left.158.657^{\circ} \mathrm{E}\right), 28 . V .1908$, A. Derzhavin (2 $\sigma^{7} \sigma^{7}$, ZISP); Krasnodar Krai: Golubitskaya $\left(45.323^{\circ} \mathrm{N} 37.274^{\circ} \mathrm{E}\right), 25$. VI.1990, E. Narchuk, ( $1{ }^{7}$, ZISP); Gelendzhik distr., Krinitsa env. (44.395 $\left.{ }^{\circ} \mathrm{N} 38.341^{\circ} \mathrm{E}\right)$, River Pshaga, 6-13.IX.2009, K. Tomkovich (1 O', ZMUM); Leningrad Oblast: Leningrad [=Saint Petersburg] env. $\left(59.878^{\circ} \mathrm{N}\right.$ $\left.29.069^{\circ} \mathrm{E}\right), 11$ and 13.VII.1984, 12.VIII.1984, M. Krivosheina (5 $\sigma^{7} \sigma^{7}$, ZMUM); Petrograd [=Saint Petersburg $\left(59.924^{\circ} \mathrm{N} 30.326^{\circ} \mathrm{E}\right)$ ], 13.IV.1919, A. Stackelberg (1 $q$, ZISP); Moscow Oblast: Dmitrov env. $\left(56.316^{\circ} \mathrm{N} 37.725^{\circ} \mathrm{E}\right)$, 2.IX.2006, N. Vikhrev $\left(1 \sigma^{7}, 1\right.$, ZMUM); North Ossetia - Alania: Sukhotskoe $\left(43.674^{\circ} \mathrm{N}\right.$ $\left.44.442^{\circ} \mathrm{E}\right), 30 \mathrm{~km} \mathrm{SW}$ of Mozdok, 3.VIII.1988, A. Ozerov (1 $\bigcirc^{7}$, ZMUM); Omsk Oblast: Cherlak-Krasny Oktyabr' env. $\left(54.133^{\circ} \mathrm{N}\right.$ $\left.75.011^{\circ} \mathrm{E}\right), 30 . V .2009$, O. Kosterin (1 9 , ZMUM); Omsk, Solenoe Lake $\left(54.885^{\circ} \mathrm{N} 73.347^{\circ} \mathrm{E}\right), 3 . \mathrm{IV}$. and 30.VI.2008, 29.VI.2013, O. Kosterin (2 $\sigma^{7} \sigma^{7}, 3$ 우, ZMUM); Omsk, Irtysh River $\left(54.99^{\circ} \mathrm{N}\right.$ 73.32 ${ }^{\circ}$ E), 22.VII.2011, O. Kosterin (1 , ZMUM); Omsk: River Irtysh left floodland $\left(54.952^{\circ} \mathrm{N} 73.355^{\circ} \mathrm{E}\right)$, reeds and salinas, 11.IV.2008, O. Kosterin (1 $0^{7}$, ZMUM); Primorsky Krai: 40 km SO of Ussuriysk $\left(43.634^{\circ} \mathrm{N} 132.222^{\circ} \mathrm{E}\right)$, 5.VIII.1983, 6.VIII.1983,

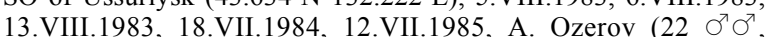
ZMUM); Vozdvizhenka (43.900 N $\left.131.943^{\circ} \mathrm{E}\right)$, garden, 26.X.1968, K. Gorodkov ( $\sigma^{7}$, ZISP); Rostov Oblast: Marga-ritovka [=Margaritovo] $\left(46.929^{\circ} \mathrm{N} 38.872^{\circ} \mathrm{E}\right), 22 . V .1953$, B. Mamaev (1 $\sigma^{\top}$, ZMUM); Yakutia: Lake Elegin, Amgino-Yakutskiy Trakt, (ca. $61.305^{\circ} \mathrm{N} 131.327^{\circ} \mathrm{E}$ ), Yakutsk Expedition RAS, 18.VIII.1925, V. Bianki (1 $\sigma^{7}$, ZISP).

ADDITIONAL MATERIAL. Kazakhstan: Chesnokovo env. $\left(51.153^{\circ} \mathrm{N} 50.453^{\circ} \mathrm{E}\right)$, Derkol River, 26.VIII.2012, K. Tomkovich ( $1 \sigma^{7}$, ZMUM); Ukraine: Alyoshki $\left(46.621^{\circ} \mathrm{N} 32.716^{\circ} \mathrm{E}\right)$, lower Dnieper, sweeping, 18.VI.1926, L. Zimin (1 ऽ, ZISP); Odessa, Kuyal'nitskiy Bay (ca. $46.565^{\circ} \mathrm{N} 30.717^{\circ} \mathrm{E}$ ), 16.IX.1938, B. Rohdendorf ( $\sigma^{7}, Z_{M U M}$; Odessa env. (ca. $\left.46.466^{\circ} \mathrm{N} 30.719^{\circ} \mathrm{E}\right)$, salt puddle near oil tank, 8.V.1926, L. Zimin (1 $\sigma^{7}$, ZMUM); Golaya Pristan' $\left(46.524^{\circ} \mathrm{N} 32.521^{\circ} \mathrm{E}\right), 18$.VII.1977, E. Klechkovskiy (1 $\sigma^{7}$, ZMUM); Chernomorskiy Nature Reserve (ca. $46.314^{\circ} \mathrm{N} 31.890^{\circ} \mathrm{E}$ ), cordon Geroyskiy, 6.VII.1977, E. Klechkovskiy (3 $\sigma^{7} \sigma^{7}$, ZMUM); Zheleznyy port env. $\left(46.124^{\circ} \mathrm{N} 32.287^{\circ} 1 \mathrm{E}\right)$, Tendrovskiy Bay, 25.VII.1977, E. Klechkovskiy (1 フ7, ZMUM); Rybach'e env. $\left(44.772^{\circ} \mathrm{N} 34.598^{\circ} \mathrm{E}\right)$, Dnepro-Bugskiy Bay, 13.VII.1977, E. Klechkovskiy ( $1 \sigma^{7}$, ZMUM).

DESCRIPTION. Head brownish-bronze or greyish pollinose except metallic shining frontal vitta and upper face, with dark greenish or bluish tinge; frontoorbital plate brownish pollinose. 3 long fronto-orbital setae, anterior interfrontal seta 2 times thinner and shorter than ocellar. Postocular setae thin and short. 

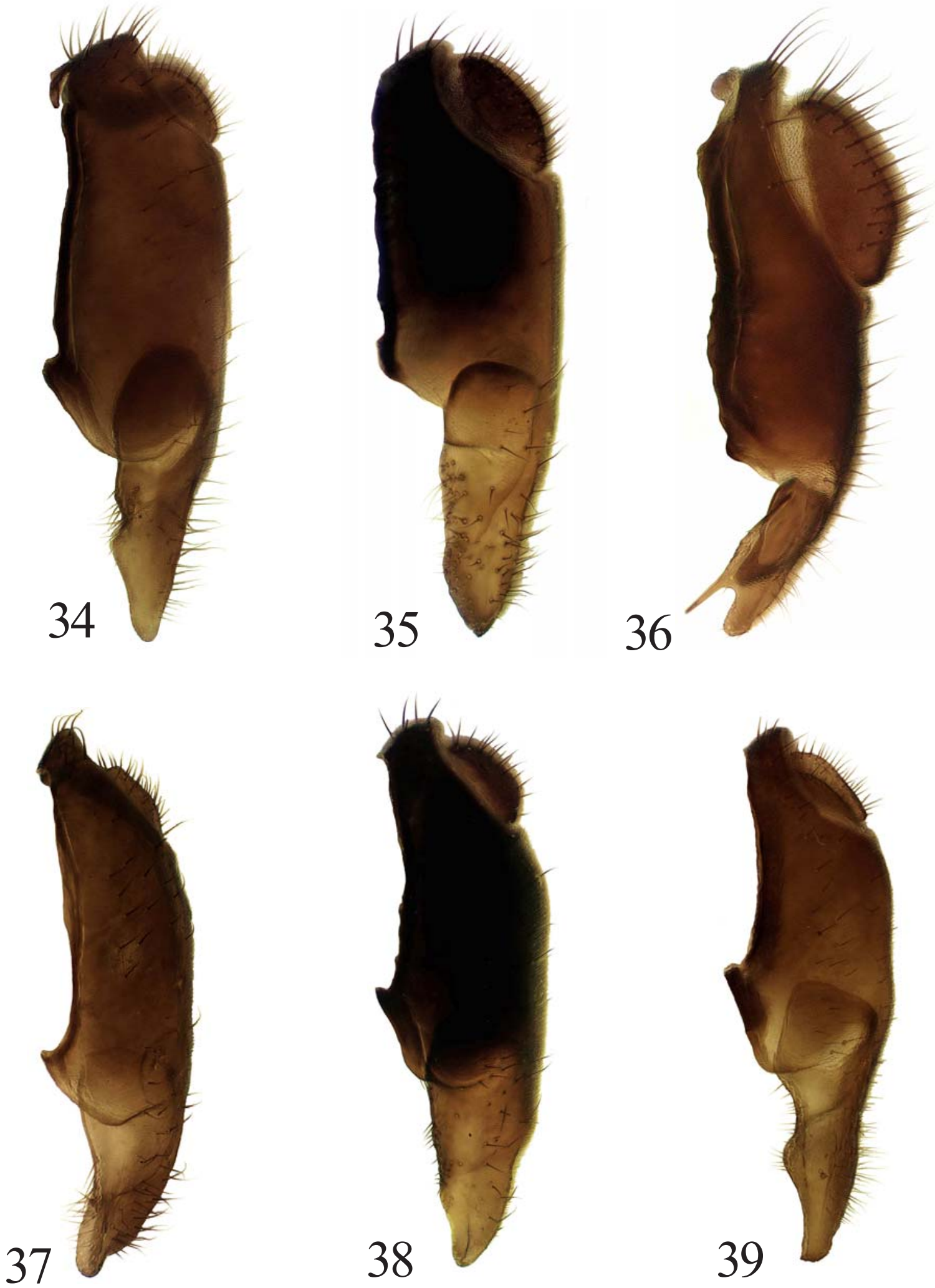

Figs 34-39. Epandrium and surstyli of Ephydra spp., lateral view: $34-$ E. afghanica Dahl; $35-$ E. attica Becker; $36-E$. glauca Meigen; $37-$ E. japonica Miyagi; $38-$ E. macellaria Egger; 39 - E. pseudomurina Krivosheina.

Рис. 34-39. Эпандриум и сурстили Ephydra spp., сбоку: $34-$ E. afghanica Dahl; $35-$ E. attica Becker; 36 - E. glauca Meigen; 37 - E. japonica Miyagi; 38 - E. macellaria Egger; 39 - E. pseudomurina Krivosheina. 

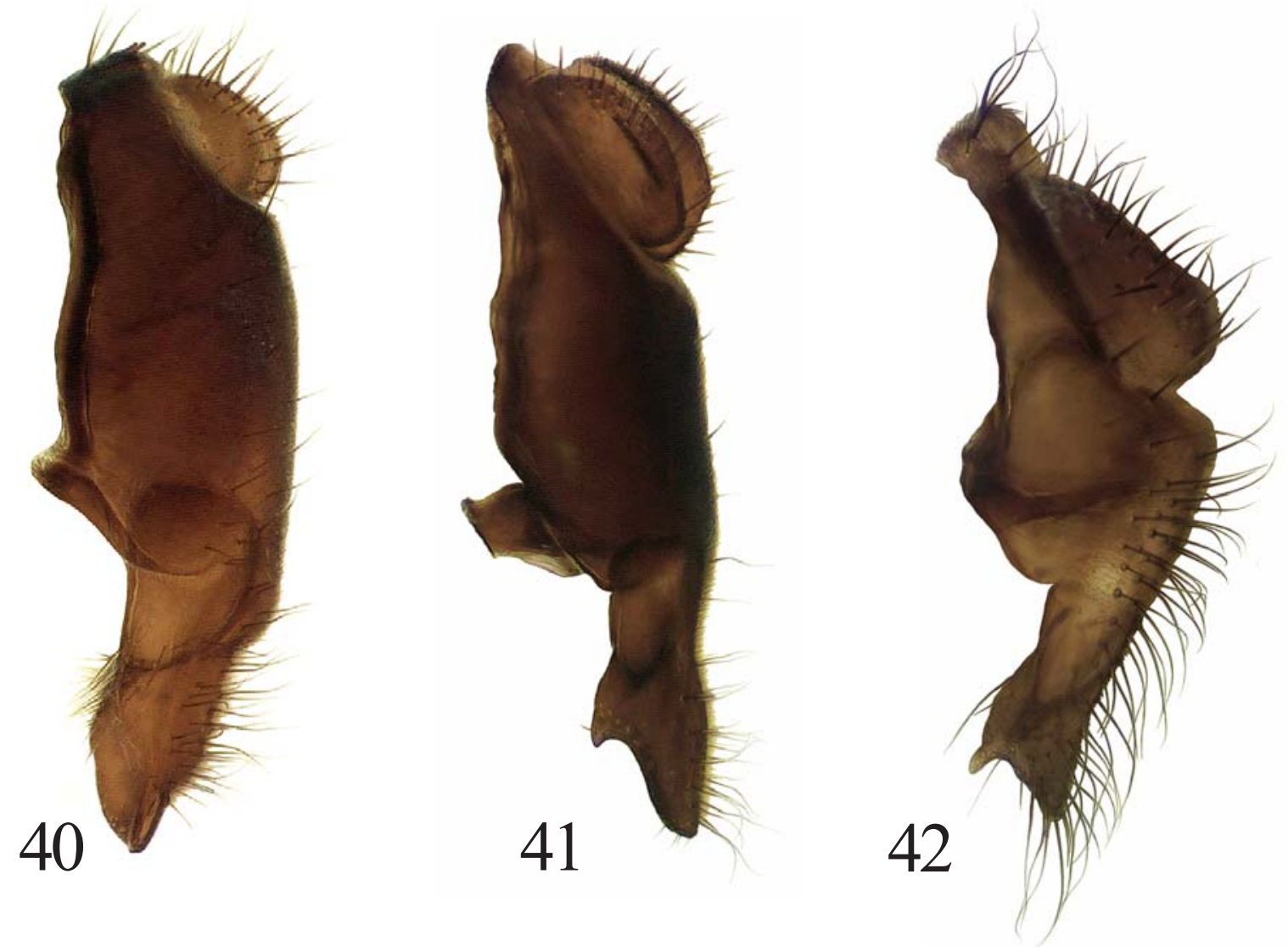

Figs 40-42. Epandrium and surstyli of Ephydra spp., lateral view: $40-$ E. riparia Fallén; $41-$ E. sholtzi Becker; $42-$ E. villosa sp.n. Рис. 40-42. Эпандриум и сурстили Ephydra spp., сбоку: $40-$ E. riparia Fallén; $41-$ E. sholtzi Becker; $42-E$. villosa sp.n.

Palpus yellow to dark brown, brownish pollinose. Antenna blackish, greyish dusted; arista pectinate on dorsal surface on basal 2/3 or more. Gena greyish dusted, parafacial bronze dusted.

Thorax black, densely brown pollinose. Scutum subshining. Acrostichals setulose in many irregular rows, intra-alars absent (Fig. 4).

Legs. Femora black densely grey dusted or yellowish grey pollinose basally, tibia and tarsi of all legs yellowish, tarsi sometimes darkened apically.

Abdomen black, subshining, grey pollinose. Tergite 5 about 2 times as long as tergite 4. Male genitalia large, as long as tergites 4+5. Epandrium oval in dorsal (posterior) view (Fig. 31); surstylus widened apically. Epandrium parallel-sided in lateral view (Fig. 40); surstylus broad and narrowed apically, with acute tip. Aedeagus with broad apex in lateral view (Fig. 13). Gonite stout at base in lateral view, with curved and slender distal part (Fig. 22).

NOTE. The species has variable coloration and it is not easy to determine it from external characters. However it is the only species with surstyli broad and significantly widened apically in dorsal view. Lateral view of surstylus resembles E. japonica, but the apex acute, not rounded.
DISRTIBUTION. Russia: Amur Oblast, Chukotka, Crimea (incl. Przhiboro \& Shadrin [2012]), Bashkiria, Bu-ryatia, Kamchatka Krai, Krasnodar Krai, Leningrad Oblast, Moscow Oblast, North Ossetia - Alania, Omsk Oblast, Primorsky Krai, Rostov Oblast, Yakutia. - Palearctic: Afghanistan, Austria, Azores, Belgium, Bulgaria, Canary Islands, China (Nei Mongol), Czech Republic, Denmark, Estonia, Finland, France, Germany, Great Britain, Hungary, Madeira Islands, Mongolia, Ne-therlands, Norway, Poland, Romania, Sweden, Tunisia, Ukraine [Mathis \& Zatwarnicki, 1995]; Kazakhstan (first record); Nearctic: Canada, United States [Mathis, Zatwarnicki, 1995].

\section{Ephydra scholtzi Becker, 1896}

Figs 2, 14, 23, 32, 41.

scholtzi Becker, 1896: 220 (Ephydra). Type-locality: "Breslau" [= Wrocław in Silesia] (Poland).

krogerusi Frey, 1930: 93 (Ephydra). Type-locality: Karelian Isthmus, Sakkola, Valkjarvi, Siikajoki, Karlo (Russia).

strandi Duda, 1942: 28 (Ephydra). Type-locality: "Nidden" [= Nida on Kuršiu Peninsula] (Lithuania).

MATERIAL. Amur Oblast: $52 \mathrm{~km}$ N of Zeya Town $\left(54.087^{\circ} \mathrm{N}\right.$ $\left.126.871^{\circ} \mathrm{E}\right)$, 5.VII.1978, A. Shatalkin (1 $\bigcirc^{7}$, ZMUM); Kamchatka Krai: Koryaki $\left(53.280^{\circ} \mathrm{N} 158.210^{\circ} \mathrm{E}\right), 50 \mathrm{~km} \mathrm{~W}$ of PetropavlovskKamchatsky, shallow, 21.VII.1978, A. Zinoviev (1 O 
Kozyrevsk $\left(56.049^{\circ} \mathrm{N} 159.865^{\circ} \mathrm{E}\right)$, shore of River Kamchatka, 27. VI.1984, N. Krivosheina (1 $\sigma^{7}$, ZMUM); Kronotskiy Nature Reserve (ca. $\left.54.749^{\circ} \mathrm{N} 160.724^{\circ} \mathrm{E}\right), 28$.VIII.1985, L. Lobkova (1 $\sigma^{7}$, ZMUM); Leningrad Oblast: Nizhnyaya Bronna $\left(59.921^{\circ} \mathrm{N}\right.$ $\left.29.621^{\circ} \mathrm{E}\right), 28$. VII.1918, A. Stackelberg (1 ㅇ, ZISP); Sestroretsk $\left(60.0916^{\circ} \mathrm{N} 29.956^{\circ} \mathrm{E}\right), 19 . \mathrm{VIII}, \mathrm{A}$. Stackelberg (1 O', 1 ㅇ, ZISP); Leningrad env. $\left(59.878^{\circ} \mathrm{N} 29.069^{\circ} \mathrm{E}\right), 13$. VII.1984, 25.VII.1984, M. Krivosheina (11 $\sigma^{\top} \sigma^{7}$, ZMUM); Nizhegorod Oblast: Dzerzhinsk $\left(56.21^{\circ} \mathrm{N} 43.62^{\circ} \mathrm{E}\right), 16-18$. VIII.2009, N. Vikhrev (1 $\sigma^{\top}$, ZMUM); Yakutia: Lensk $\left(60.727^{\circ} \mathrm{N} 114.943^{\circ} \mathrm{E}\right), 14 . I X .1987, \mathrm{~K}$. Gorodkov (1 $\sigma^{7}$, ZISP);

ADDITIONAL MATERIAL. Kazakhstan: sands Kyzylkum, 30 $\mathrm{km} \mathrm{S}$ of Samarskoe (ca. $48.716^{\circ} \mathrm{N} 83.352^{\circ} \mathrm{E}$ ), 3-4.VIII.1987, E. Nartshuk ( $\sigma^{7}, 6$ 웅, ZISP); Mongolia: Central aimag [=Töv aimag], $20 \mathrm{~km} \mathrm{~S}$ of Ulan-Bator (ca. $47.717^{\circ} \mathrm{N} 106.925^{\circ} \mathrm{E}$ ), 2.VII 1967, V. Zaitsev (1 $\sigma^{T}$, ZISP); Tadjikistan: Stalinabad [=Dushanbe $\left.\left(38.569^{\circ} \mathrm{N} 68.774^{\circ} \mathrm{E}\right)\right]$, 8.VII.1945, V. Gussakovskiy (1 $\sigma^{7}$ ZISP).

DESCRIPTION. Head (Fig. 2) silvery-grey pollinose except metallic shining frontal vitta and upper face, with bluish-greenish tinge; fronto-orbital plate grey pollinose. 3 long fronto-orbital setae, anterior interfrontal seta strong, a little shorter, than ocellar. Postocular setae short and thin. Palpus grey. Antenna blackish, greyish dusted; arista very short pubescent on dorsal surface on basal 1/2 (Fig. 2). Gena and parafacial silvery-grey dusted.

Thorax black, densely grey pollinose. Scutum metallic subshining with brownish stripes. Acrostichals setulose in two rows, intra-alars 1+2 (often weak).

Legs. Femora and tibia black, except yellow bases of tibiae, densely greyish dusted; tarsi of all legs yellowish, tarsomeres 4 and 5 darkened.

Abdomen black with greenish tinge, golden-grey pollinose, subshining. Tergite 5 approximately equal in length to tergite 4 . Male genitalia small, as long as tergite 5. Epandrium elongate in dorsal (posterior) view (Fig. 32), wider in cercal half; surstylus broad and oval. Epandrium parallel-sided in lateral view (Fig. 41); surstylus is continuation of epandrium, with short median lobe. Aedeagus relatively wide with pointed tip in lateral view (Fig. 14). Gonite with broad basal part and slender pointed tip in lateral view (Fig. 23).

NOTE. The species is easily determined by the following combination of characters: acrostichal setae in two rows and postocular setae short and thin. Additional diagnostic characters may be silvery grey coloration of face and dark femora and tibiae.

DISRTIBUTION. Russia: Amur Oblast, Kamchatka Krai, Karelia, Leningrad Oblast, Nizhegorod Oblast, Yakutia. - Palearctic: Bulgaria, Denmark, Finland, France, Germany, Lithuania, Netherlands, Poland, Romania, Sweden [Mathis, Zatwarnicki, 1995]; Tadjikistan (first record), Kazakhstan (first record), Mongolia (first record).

\section{Ephydra villosa Krivosheina et Ozerov, sp.n. Figs 15, 24, 33, 42.}

MATERIAL. Holotype $\sigma^{r}$, Russia: Irkutsk Oblast, Baikal Lake, Sakhyurta env., MRS [=river station Malomorskaya) $\left(53.017^{\circ} \mathrm{N}\right.$ $\left.106.883^{\circ} \mathrm{E}\right)$ ], 19.VII.1962, Gorodkov (ZISP). Paratype $\sigma^{7}$ with same label (ZMUM).

\section{DESCRIPTION. Medium sized flies, body length} $5-5.5 \mathrm{~mm}$.

Head golden-brown pollinose except metallic shining frontal vitta and upper face, with greenish tinge; fronto-orbital plate brownish pollinose. 3 long frontoorbital setae, anterior interfrontal strong, about $1 / 2$ of ocellar. Postocular setae strong. Palpus dark grey. Antenna blackish, brownish dusted; arista short pubescent on dorsal surface on basal $1 / 2$. Gena and parafacial golden dusted.

Thorax black, brownish pollinose. Scutum subshining with bluish-greenish tinger. Acrostichals setulose in two rows, intra-alars $2+2$.

Legs. Femora and tibia black, densely greyish dusted; tarsi of all legs yellowish, but tarsomeres 4 and 5 darkened.

Abdomen black, grey pollinose, subshining. Tergite 5 approximately equal in length to tergite 4 . Male genitalia small, as long as tergite 5. Epandrium oval in dorsal (posterior) view (Fig. 33), wider in cercal part, covered with long setae; surstyli relatively broad and curved, covered with many long setae. Epandrium curved in lateral view (Fig. 42); surstylus is at obtuse angle to epandrium, with short subapical tooth. Aedeagus long and narrow with pointed tip in lateral view (Fig. 15). Gonite with broad basal part and slender pointed tip in lateral view (Fig. 24).

NOTE. The species is close to E. tibetensis Wirth, 1975 , differing from it in extremely developed setae and hairs on body and the structure of male genitalia; surstylus with well separated subapical tooth in lateral view, aedeagus with long apical narrow part, gonite significantly narrowed apically and curved at the very tip. From E. scholtzi the new species differs in strong postocular setae and from E. glauca in weaker pollinosity and the presence of subshining surfaces on upper face, scutum and abdomen. From both species it differs in the development of 2+2 intra-alar setae.

DISRTIBUTION. Russia: Irkutsk Oblast.

\section{Key to SPECIES OF the Genus EPHydRA OF RUSSiA}

1. Anterior acrostichal setae in two distinct rows (e.g., Fig. 3 ); tarsal claws generally nearly as long as tarsomere 5; male aedeagus nearly straight, lacking recurved basal process (e.g., Fig. 9) (The glauca group) .................. 2

- Anterior acrostichal setae in many irregular rows (e.g., Fig. 4); tarsal claws generally no more than half of tarsomere 5; male aedeagus with strongly recurved basal process (e.g., Fig. 8) (The riparia group) ..................... 3

2. Postocular setae short and thin (Fig. 2). Surstylus in dorsal view elongate-oval (Fig. 32), in lateral view as in Fig. 41. Aedeagus (Fig. 14). Gonite (Fig. 23)

E. scholtzi Becker

- Postocular setae strong, well developed (Fig. 1) .............. 4

3 . Body densely grey pollinose, upper face, scutum and abdomen not subshining, 1+2 intra-alar setae present. Surstylus in dorsal view wide basally and thin apically (Fig. 27), in lateral view as in Fig. 36. Aedeagus (Fig. 9). Gonite (Fig. 18) .............................. E. glauca Meigen

- Body golden-grey pollinose, upper face, scutum and abdomen subshining, $2+2$ intra-alar setae present. Surstylus 
in dorsal view curved and covered with many setae (Fig. 33), in lateral view as in Fig. 42. Aedeagus (Fig. 15). Gonite (Fig. 24) E. villosa sp.n. 4. Tergite 5 of male 2 times or more as long as tergite 4 ....... 5 - Tergite 5 of male 1.3-1.7 times but no more than 2 times as long as tergite 4 ......

5. Legs yellow. Surstylus in dorsal view very thin and curve (Fig. 25), in lateral view as in Fig. 34. Aedeagus (Fig. 7). Gonite (Fig. 16) E. afghanica Dahl

- Femora black or yellow, grey dusted

6. Anterior interfrontal seta $1 / 2$ as long as ocellar. Abdomen unicolor. Surstylus in dorsal view widened apically (Fig. 31), in lateral view pointed apically (Fig. 40). Aedeagus (Fig. 13). Gonite (Fig. 22)

E. riparia Fallén

- Anterior interfrontal seta $1 / 3$ as long as ocellar. Male abdominal tergite 5 often golden, contrasting to grey coloration of other tergites. Surstylus in dorsal view straight and not widened (Fig. 28), in lateral view broadly rounded and curved (Fig. 37). Aedeagus (Fig. 10). Gonite (Fig. 19)

E. japonica Miyagi

7. Palpus yellow-grey to dark grey. Tergite 5 of male 1.31.5 as long as tergite 4 . Surstylus in dorsal view thin and straight, not covered with long setae (Fig. 29), in lateral view as in Fig. 38. Aedeagus (Fig. 11). Gonite (Fig. 20) E. macellaria Egger

- Palpus yellow. Tergite 5 of male 1.5-2.0 as long as tergite

8. Femora yellow, greyish dusted, or yellow. Surstylus in dorsal view broad and rounded apically, covered with short setae (Fig. 30), in lateral view asymmetrical with median lobe (Fig. 39). Aedeagus (Fig. 12). Gonite (Fig. 21) E. pseudomurina Krivosheina

- Femora yellow or black, grey dusted. Surstylus in dorsal view thin, parallel, widened at the most apex, covered with long setae (Fig. 26), in lateral view broad, symmetrical, pointed at apex (Fig. 35). Aedeagus (Fig. 8). Gonite (Fig. 17) E. attica Becker

Acknowledgements. We are very grateful to Dr. Olga Ovchinnikova and Mrs. Galina Suleymanova for the help during our work in ZISP with the material of Ephydra. We also thank Mr.Dmitry Gavryushin for valuable comments and text editing.

The investigation was fulfilled within the State project of the Institute of Ecology and Evolution, Russian Academy of Sciences (M.G. Krivosheina) and within the framework of the State project No 121032300105-0 of Lomonosov Moscow State University (A.L. Ozerov).

\section{References}

Becker Th. 1896. Dipterologische Studien IV. Ephydridae // Berliner Entomologische Zeitschrift. Bd.41. H.2. S.91-76.

Beschovski V.L. 2009. [Shore flies (Insecta: Diptera: Ephydridae, Tethinindae, Canacidae)] // Fauna of Bulgaria. Vol. 28. Sofia: Academical Press "Prof. Marin Drinov". 440 p. [In Bulgarian]

Cumming J.M., Wood D.M. 2009. Adult morphology and terminology // Brown et al. (eds.). Manual of Central American Diptera. Vol.1. Ottawa: National Research Council Press. P.9-50.

Dahl R.G. 1961. Ephydridae (Diptera Brachycera) from Afghanistan, Contribution à l'étude de la faune d'Afghanistan $15 / /$ Entomologisk Tidskrift. Vol.82. No.1-2. P.87-90.

Duda O. 1942. Neue oder ungenügend bekannte Zweiflügler der paläarktischen Region aus meiner Sammlung. 2. Fortsetzung // Deutsche Entomologische Zeitschrift. H.1-4. S.1-39.
Egger J. 1862. Dipterologische Beiträge. Fortsetzung der Beschreibungen neuer Dipteren // Verhandlungen der kaiser-lich-königlichen Zoologisch-Botanischen Gesellschaft in Wien. Bd.12. S.777-784.

El-Hawagry M.S., Zatwarnicki T., Ayman M.E. 2018. Catalogue of the Egyptian Ephydroidea (Diptera: Schizophora: Acalyptratae) // Zootaxa. Vol.4444. No.3. P.201-246. doi.org/10. 11646/zootaxa.4444.3.1

El-Moursy A.A., Negm F.H., El-Hawagry M.S., Ayman M. E. 2006. Review of the subfamily Ephydrinae (Ephydridae, Diptera) from Egypt, with a description of a new species // Bulletin of the Entomological Society of Egypt. Vol.83. P.7197.

Fallén C.F. 1810. Specimen entomologicum novam Diptera disponendi methodum exhibens. Berlingianis. Lundae $[=$ Lund $]$. $26 \mathrm{p}$.

Fallén C.F. 1813. Beskrifning öfver några i Sverige funna Vattenflugor (Hydromyzides) // Kongliga Vetenskaps-Aca-demiens Handling. Ser.3. S.240-257.

Frey R. 1909. Mitteilungen über finnländische Dipteren // Acta Societatis pro Fauna et Flora Fennica. Bd.31. H.9. S.1-23.

Frey R. 1930. Neue Diptera brachycera aus Finnland und angrenzenden Ländern // Notulae Entomologicae. No.10. S.82-94.

Giordani Soika A.G. 1960. Beschreibung einer neu entdeckten Art der Ephydriden-Gattung Ephydra Fallén (Diptera) // Deutsche Entomologische Zeitschrift. Bd.7. H.4-5. S.456457.

Heyden C.H.G. von. 1843. Ueber Insekten die an den Salinen leben // Stettiner Entomologische Zeitung. Bd.4. H.8. S.227229.

Heyden C.H.G. von. 1844. Ferne Nachrichten über Insecten der Salinen // Stettiner Entomologische Zeitung. Bd.5. H.6. S.202205.

Hu X., Jang D. 2001. New species and new records of the genus Ephydra Fallén (Diptera, Ephydridae) from China // Studia dipterologica. Bd.8. H.2. S.529-537.

Krivosheina M.G. 1983. [A new species of the fly genus Ephydra Fll. (Diptera, Ephydridae) from Uzbekistan] // Entomologicheskoe obozrenie. Vol.62. No.2. P.367-370 [in Russian].

Krivosheina M.G. 1985. [Ephydridae (Diptera) new for the USSR] // Entomologicheskoe obozrenie. Vol.64. No.2. P.419-422 [in Russian].

Krivosheina M.G. 1986. [To the biology of the shore-flies (Diptera, Ephydridae) of anthropogenic landscapes of the desert zone] // Nauchnye Doklady Vysschei Shkoly. Biologicheskie Nauki. No.5. P.40-43 [in Russian].

Krivosheina M.G. 1987. [On the fauna of shore-flies (Diptera, Ephydridae) of the Far East] // Lehr P.A., Kanyukova E.V. (eds.). Taksonomia nasekhomych Sibirii i Dal'nego Vostoka SSSR. Vladivostok. P.116-123 [in Russian].

Krivosheina M.G. 2003. To the biology of flies of the genus Ephydra Fallén, 1810, with the descriptions of larvae of seven Palaearctic species (Diptera: Ephydridae) // Russian Entomological Journal. Vol.12. No.1. P.79-86.

Krivosheina M.G. 2010. Shore-flies (Diptera, Ephydridae) of Lake Baskunchak // Euroasian Entomological Journal. Vol.9. No.4. P.666-667.

Krivosheina M.G. 2014. [To the fauna of Crimean shore-flies (Diptera: Ephydridae)] // Eversmannia. Vol.39. P.42-44 [in Russian].

Krivosheina M.G., Ozerov A.L. 2020. A Review of the Shore Fly Genus Setacera Cresson, 1930 (Diptera, Ephydridae) in the fauna of Russia // Entomological Review. Vol.10. No.8. P.11901200. doi: $10.1134 / \mathrm{S} 0013873820080114$

Mathis W.N., Marioni L. 2016. Revision of Ephydrini Zetterstedt (Diptera: Ephydridae) from the Americas south of the United States // Zootaxa. Vol.4116. No.1. P.1-110. doi.org/10.11646/ zootaxa.4116.1.1

Mathis W.N., Zatwarnicki Ò. 1995. World Catalog of Shore Flies (Diptera: Ephydridae) // Memoires of Entomology, International. Vol.4. P.1-423.

McAlpine J.F. 1981. Morphology and terminology-adults // McAlpine J.F., Peterson B.V., Shewell G.E., Teskey H.J., Vockeroth J.R., Wood D.M. (Coordinators). Manual of Nearctic Diptera. Vol.2. 
Ottawa: Research Branch. Agriculture Canada. Monograph 27. P.9-63.

Meigen J.W. 1830. Systematische Beschreibung der bekannten europäischen zweiflügeligen Insekten. 6. Schulz-Wundermann. Hamm. $401 \mathrm{~S}$.

Miyagi I. 1966. Descriptions of three new species of Ephydridae, with one unrecorded species from Japan (Diptera, Ephydridae) // Kontyû. Vol.34. No.2. P.136-140.

Nartshuk E.P. 1970. [Fam. Ephydridae - shore flies] // Bei-Bienko G.Ya. (ed.). Opredelitel' nasekomykh Evropeiskoi chast SSSR. Leningrad: Nauka. P.363-389 [in Russian].

Przhiboro A., Shadrin N. 2012. Mass occurrence of flies of the genus Ephydra (Diptera: Ephydridae) at the coastline zone of hypersaline coastal lagoons in the Eastern Crimea // Marine ekological journal. Vol.11. No.1. P.24.
Shayhutdinova A.A., Krivosheina M.G. 2020. Invertebrates of hypergaline reservoirs of the Orenburg Region (Russia) // Russian Entomological Journal. Vol.20. No.3. P.337-342.

Stuckenberg B.R. 1999. Antennal evolution in the Brachycera (Diptera), with a reassessment of terminology relating to the flagellum // Studia Dipterologica. Bd.6. S.33-48.

Wirth W.W. 1975. A revision of the brine flies of the genus Ephydra of the Old World (Diptera: Ephydridae) // Entomologica Scandinavica. Vol.6. No.1. P.11-44.

Zatwarnicki T., Kahanpää J. 2014. Checklist of the family Ephydridae of Finland (Insecta, Diptera) // ZooKeys. Vol.441. P.339346. doi: 10.3897/zookeys.441.7448

Zetterstedt J.W. 1846. Diptera scandinaviae disposita et descripta. Tomus quintus. Officina Lundbergiana. Lundae [= Lund]. P.1739-2162. 